TRANSACTIONS OF THE

AMERICAN MATHEMATICAL SOCIETY

Volume 357, Number 7, Pages 2627-2664

S 0002-9947(05)03781-5

Article electronically published on March 1, 2005

\title{
ESTIMATES OF THE DERIVATIVES FOR PARABOLIC OPERATORS WITH UNBOUNDED COEFFICIENTS
}

\author{
MARCELLO BERTOLDI AND LUCA LORENZI
}

\begin{abstract}
We consider a class of second-order uniformly elliptic operators $\mathcal{A}$ with unbounded coefficients in $\mathbb{R}^{N}$. Using a Bernstein approach we provide several uniform estimates for the semigroup $T(t)$ generated by the realization of the operator $\mathcal{A}$ in the space of all bounded and continuous or Hölder continuous functions in $\mathbb{R}^{N}$. As a consequence, we obtain optimal Schauder estimates for the solution to both the elliptic equation $\lambda u-\mathcal{A} u=f(\lambda>0)$ and the nonhomogeneous Dirichlet Cauchy problem $D_{t} u=\mathcal{A} u+g$. Then, we prove two different kinds of pointwise estimates of $T(t)$ that can be used to prove a Liouville-type theorem. Finally, we provide sharp estimates of the semigroup $T(t)$ in weighted $L^{p}$-spaces related to the invariant measure associated with the semigroup.
\end{abstract}

\section{INTRODUCTION}

In recent years much attention has been paid to the uniformly elliptic operator

$$
\mathcal{A}=\sum_{i, j=1}^{N} q_{i j}(x) D_{i j}+\sum_{j=1}^{N} b_{j}(x) D_{j},
$$

with unbounded coefficients in $\mathbb{R}^{N}$. The interest for operators with unbounded coefficients is due to the fact that they arise naturally in the theory of Markov processes. Actually these operators have very interesting properties, quite different from those enjoyed by elliptic operators with bounded coefficients.

If $f$ is continuous and bounded in $\mathbb{R}^{N}\left(f \in C_{b}\left(\mathbb{R}^{N}\right)\right.$ for short $)$, under suitable hypotheses the Cauchy problem

$$
\left\{\begin{array}{lr}
D_{t} u(t, x)=\mathcal{A} u(t, x), \quad t>0, & x \in \mathbb{R}^{N}, \\
u(0, x)=f(x), & x \in \mathbb{R}^{N},
\end{array}\right.
$$

has a unique bounded solution. Moreover, there exists a semigroup $\{T(t)\}_{t \geq 0}$ of linear operators in $C_{b}\left(\mathbb{R}^{N}\right)$ such that $u(t, x)=(T(t) f)(x)$. In general, $T(t)$ is neither strongly continuous nor analytic (see [14]).

Received by the editors July 7, 2003.

2000 Mathematics Subject Classification. Primary 35B45; Secondary 35B65, 35K10, 47D06.

Key words and phrases. Elliptic and parabolic operators with unbounded coefficients in $\mathbb{R}^{N}$, Markov semigroups, uniform and pointwise estimates, optimal Schauder estimates.

This work was partially supported by the research project "Equazioni di evoluzione deterministiche e stocastiche" of the Ministero dell'Istruzione, dell'Università e della Ricerca (M.I.U.R.) and by the European Community's Human Potential Programme under contract HPRN-CT-200200281 "Evolution Equations".

(C)2005 American Mathematical Society Reverts to public domain 28 years from publication 
The problem of estimating the derivatives of $T(t) f$ has already been studied in literature by both analytic ([2, 4, 6, 12]) and probabilistic methods ([5, 19]).

In this paper we prove both uniform and pointwise estimates for the first-, secondand third-order derivatives of $T(t) f$. In section 3 we prove uniform estimates, namely we show that for any $\omega>0$ and any $k, l \in \mathbb{N}, 0 \leq k \leq l \leq 3$, there exists a positive constant $C_{k, l}=C_{k, l}(\omega)$ such that

$$
\|T(t) f\|_{C_{b}^{l}\left(\mathbb{R}^{N}\right)} \leq C_{k, l} t^{-(l-k) / 2} e^{\omega t}\|f\|_{C_{b}^{k}\left(\mathbb{R}^{N}\right)}, \quad \forall f \in C_{b}^{k}\left(\mathbb{R}^{N}\right), t>0 .
$$

To prove (1.2) we use the Bernstein method and approximate $T(t) f$ by solutions of Cauchy problems in bounded domains. We assume dissipativity-type and growth conditions on the coefficients of $\mathcal{A}$. We notice that some dissipativity condition is necessary, because in general estimate (1.2) fails; see [4.

Then, using interpolation arguments, we obtain similar estimates for $T(t) f$ in Hölder spaces. Namely, we extend estimate (1.2) to the case when $k, l \in \mathbb{R}_{+}$, $0 \leq k \leq l \leq 3$. This allows us to prove optimal Schauder estimates for the solution of the nonhomogeneous Dirichlet Cauchy problem associated with (1.1) as well as for the elliptic equation $\lambda u-\mathcal{A} u=f(\lambda>0)$.

In section 4 we consider the following pointwise estimates for the derivatives of $T(t) f$ : first we prove that for any $k=1,2,3$ and any $p \in(1,+\infty)$, there exists a constant $\sigma_{k, p} \in \mathbb{R}$ such that

$$
\left|\left(D^{k} T(t) f\right)(x)\right|^{p} \leq e^{\sigma_{k, p} t}\left(T(t)\left(\left(|f|^{2}+\ldots+\left|D^{k} f\right|^{2}\right)^{\frac{p}{2}}\right)\right)(x), \quad \forall t>0, x \in \mathbb{R}^{N},
$$

for all $f \in C_{b}^{k}\left(\mathbb{R}^{N}\right)$. Consequently we deduce the second pointwise estimate

$$
\begin{aligned}
\left|\left(D^{k} T(t) f\right)(x)\right|^{p} \leq & \left(\frac{\sigma_{k, \min \{p, 2\}}}{1-e^{-\sigma_{k, \min \{p, 2\}} t}} \phi_{k, \min \{p, 2\}}(t)\right)^{\max \left\{1, \frac{p}{2}\right\}} \\
& \quad \times\left(T(t)\left(\left(|f|^{2}+\ldots+\left|D^{k-1} f\right|^{2}\right)^{\frac{p}{2}}\right)\right)(x),
\end{aligned}
$$

for any $(t, x) \in \mathbb{R}_{+} \times \mathbb{R}^{N}$, any $f \in C_{b}^{k-1}\left(\mathbb{R}^{N}\right)$ and any $p>1$, where $\phi_{k, r} \in$ $C([0,+\infty))$ is a suitable function which behaves as $t^{1-r / 2}$ near 0 and may grow exponentially at infinity (see (4.16) ). Then, taking the semigroup property into account, from (1.4) one readily obtains the estimate

$$
\left|\left(D^{k} T(t) f\right)(x)\right|^{p} \leq C_{k, p} \frac{e^{\omega_{k, p} t}}{t^{p k / 2}}\left(T(t)|f|^{p}\right)(x), \quad t>0, x \in \mathbb{R}^{N},
$$

for any $f \in C_{b}\left(\mathbb{R}^{N}\right)$, any $p>1$ and some constants $C_{k, p}>0$, blowing up as $p$ tends to 1 , and $\omega_{k, p} \in \mathbb{R}$.

In the particular case when $q_{i j}(x)=\delta_{i j}$, i.e. when $\mathcal{A}=\Delta+\sum b_{i}(x) D_{i}$, we also prove estimate (1.3) for $p=1$. On the contrary, estimate (1.5) cannot, in general, be extended to the case when $p=1$. Counterexamples are easily obtained in the simple case $\mathcal{A}=\Delta$ (see [14]).

Such pointwise estimates are typical for transition semigroups of Markov processes, and they have already been studied for the first-order derivatives $(k=1)$; see [1, 2], 3].

In the case when $\omega_{1, p} \leq 0$, estimate (1.5) with $k=1$ allows us to obtain a Liouville-type theorem, namely, in such a situation we can show that, if $\mathcal{A} u=0$, then $u$ is constant. If $\omega_{1, p}>0$, in general, such a result fails. Counterexamples are also given in [18] in the one-dimensional case. 
In the last section we derive from (1.5) similar estimates for the derivatives of the extension of $T(t) f$ to the $L^{p}$-spaces related to the invariant measure $\mu$ associated with the semigroup. To be more precise, we show that, for any $\omega>0$, any $k=1,2,3$ and any $p \in(1,+\infty)$, there exists a positive constant $C_{k, p}=C_{k, p}(\omega)$ such that

$$
\left\|D^{k} T(t) f\right\|_{p} \leq C_{k, p} \frac{e^{\omega_{k, p} t}}{t^{k / 2}}\|f\|_{p}, \quad t>0
$$

for all $f \in L^{p}\left(\mathbb{R}^{N}, \mu\right)$, where $\|\cdot\|_{p}$ denotes the norm of $L^{p}\left(\mathbb{R}^{N}, \mu\right)$.

The characterization of the domain $D\left(A_{p}\right)$ of the realization of $A_{p}$ in $L^{p}\left(\mathbb{R}^{N}, \mu\right)$ is still an open problem. There are just a few results in the particular case when the drift term is a gradient (see [16]) or $\mathcal{A}$ is an Ornstein-Uhlenbeck operator (see 15]). In such a situation, the invariant measure can be explicitly determined, and things are easier. In general, the invariant measure is unknown and there are only a few results on the regularity and behaviour at infinity of its density $d \mu / d x$, which do not allow us to perform the same technique as in [16] to characterize $D\left(A_{p}\right)$.

One of the main consequence of (1.6), with $k=1$, is that it allows us to give a partial characterization of $D\left(A_{p}\right)$. Indeed, using a standard technique relying on the representation of the resolvent $R\left(\lambda, A_{p}\right) f$, at $x \in \mathbb{R}^{N}$, as the Laplace transform of the function $t \mapsto(T(t) f)(x)$, we show that if $f \in D\left(A_{p}\right)$, then the first distributional derivatives $D_{k} f, k=1, \ldots, N$, belong to $L^{p}\left(\mathbb{R}^{N}, \mu\right)$.

Notation. Throughout the paper we denote by $B(R)$ the open ball in $\mathbb{R}^{N}$ with center at $x=0$ and radius $R$, and by $\bar{B}(R)$ its closure in $\mathbb{R}^{N}$. For any $a, b \in \mathbb{R}$, we denote by $a \vee b$ and $a \wedge b$, respectively, the maximum and the minimum between $a$ and $b$. When $b=0$ we set $a^{+}=a \vee 0$. For any multi-index $\alpha=\left(\alpha_{1}, \ldots, \alpha_{n}\right)$, any open set $\Omega \subset \mathbb{R}^{N}$ and any smooth function $f: \Omega \rightarrow \mathbb{R}$ we set $D^{\alpha} f=D_{x_{1}}^{\alpha_{1}} \cdots D_{x_{N}}^{\alpha_{N}} f$. Similarly, for any $k \in \mathbb{N}$ and any $x \in \Omega$ we set $\left|D^{k} f(x)\right|^{2}=\sum_{|\alpha|=k}\left|D^{\alpha} f(x)\right|^{2}$.

\section{FunCtion SPACES AND PRELIMINARIES}

In this section we define the function spaces we deal with throughout this paper and we collect all the needed preliminary results.

Definition 2.1. We denote by $C_{b}\left(\mathbb{R}^{N}\right)$ the space of all bounded and continuous functions $f: \mathbb{R}^{N} \rightarrow \mathbb{R}$ endowed with the sup-norm. Moreover, for any $k \in \mathbb{R}_{+}$, we denote by $C_{b}^{k}\left(\mathbb{R}^{N}\right)$ the spaces of all continuously differentiable up to the $[k]$-order functions $f$ such that $D^{\alpha} f \in C_{b}\left(\mathbb{R}^{N}\right)$ for any $|\alpha| \leq[k]$ ([k] denoting the integer part of $k$ ) and $D^{\alpha} f$ is $(k-[k])$-Hölder continuous. We endow $C_{b}^{k}\left(\mathbb{R}^{N}\right)$ with the norm

$$
\|f\|_{C_{b}^{k}\left(\mathbb{R}^{N}\right)}=\sum_{|\alpha| \leq[k]}\left\|D^{\alpha} f\right\|_{\infty}+\sum_{|\alpha|=[k]}\left[D^{\alpha} f\right]_{C^{k-[k]}\left(\mathbb{R}^{N}\right)}, \quad \forall f \in C_{b}^{k}\left(\mathbb{R}^{N}\right),
$$

where

$$
[g]_{C^{k-[k]}\left(\mathbb{R}^{N}\right)}=\sup _{x, y \in \mathbb{R}^{N}, x \neq y} \frac{|g(x)-g(y)|}{|x-y|^{k-[k]}}, \quad \forall g \in C^{k-[k]}\left(\mathbb{R}^{N}\right) .
$$

For any open set $\Omega \subset \mathbb{R}^{N}$ and any $k \in[0,+\infty], C_{0}^{k}(\Omega)$ denotes the subset of $C^{k}\left(\mathbb{R}^{N}\right)$ of all the functions $f$ compactly supported in $\Omega$.

By $C_{\text {loc }}^{k}\left(\mathbb{R}^{N}\right), k \in \mathbb{R}_{+}$, we denote the set of all the functions $f: \mathbb{R}^{N} \rightarrow \mathbb{R}$ which belong to $C^{k}(K)$ for any compact set $K \subset \mathbb{R}^{N}$. Similarly,

$$
C_{l o c}^{1+\alpha / 2,2+\alpha}\left((0,+\infty) \times \mathbb{R}^{N}\right)
$$


denotes the space of the functions $u$ which are once continuously differentiable with respect to time and twice continuously differentiable with respect to the space variables in $(0,+\infty) \times \mathbb{R}^{N}$ and such that for any compact set $D \subset(0,+\infty) \times \mathbb{R}^{N}$, $D_{t} u, D_{x}^{\beta} u(|\beta| \leq 2)$ are $\alpha$-Hölder continuous in $D$ with respect to the parabolic distance $d((t, x),(s, y))=\left(|t-s|+|x-y|^{2}\right)^{1 / 2}$. For any $k \in \mathbb{N}$ and any compact set $D \subset \mathbb{R} \times \mathbb{R}^{N}, C^{1+\alpha / 2,2+k+\alpha}(D)$ denotes the set of all the $u$ 's which admit space derivatives up to the $k$-th order in $C^{1+\alpha / 2,2+\alpha}(D)$.

For any positive measure $\mu$ we denote by $L^{p}\left(\mathbb{R}^{N}, \mu\right)(p \in[1,+\infty))\left(L_{\mu}^{p}\right.$ for short $)$ the $L^{p}$-space related to the measure $\mu$ and we endow it with the usual norm.

Finally, we denote by $W^{k, p}\left(\mathbb{R}^{N}, \mu\right)(k \in \mathbb{N}, p \in[1,+\infty))\left(W_{\mu}^{k, p}\right.$ for short) the space of all the functions $f: \mathbb{R}^{N} \rightarrow \mathbb{R}$ admitting weak derivatives up to the $k$-order in $L_{\mu}^{p}$. We endow it with the norm

$$
\|f\|_{W_{\mu}^{k, p}}=\sum_{|\alpha| \leq k}\left\|D^{\alpha} f\right\|_{L_{\mu}^{p},} \quad \forall f \in W_{\mu}^{k, p} .
$$

We now recall some basic results on the Cauchy problem

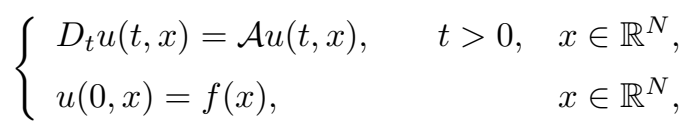

where $f \in C_{b}\left(\mathbb{R}^{N}\right)$. Under the following hypothesis:

H1): $q_{i j}, b_{j} \in C_{l o c}^{\delta}\left(\mathbb{R}^{N}\right)$ for some $\delta \in(0,1)$ and $q_{i j}(x)=q_{j i}(x)$ for any $i, j=$ $1, \ldots, N$ and any $x \in \mathbb{R}^{N}$, and

$$
\sum_{i, j=1}^{N} q_{i j}(x) \xi_{i} \xi_{j} \geq \nu(x)|\xi|^{2}, \quad \forall \xi, x \in \mathbb{R}^{N},
$$

for some function $\nu: \mathbb{R}^{N} \rightarrow \mathbb{R}$ such that $\inf _{x \in \mathbb{R}^{N}} \nu(x)=\nu_{0}>0$,

problem (2.1) admits a classical solution

$$
u \in C\left([0,+\infty) \times \mathbb{R}^{N}\right) \cap C_{l o c}^{1+\delta / 2,2+\delta}\left((0,+\infty) \times \mathbb{R}^{N}\right)
$$

satisfying

$$
|u(t, x)| \leq\|f\|_{\infty}, \quad t>0, x \in \mathbb{R}^{N} .
$$

Without any additional assumption, in general the function $u$ is not the unique classical bounded solution to problem (2.1) (see [9] 13] for examples of nonuniqueness). If we assume the further condition

H2): there exists $\lambda>0$ and a function $\varphi \in C^{2}\left(\mathbb{R}^{N}\right)$ such that

$$
\lim _{|x| \rightarrow+\infty} \varphi(x)=+\infty \quad \text { and } \quad \sup _{x \in \mathbb{R}^{N}}(\mathcal{A} \varphi(x)-\lambda \varphi(x))<+\infty,
$$

then the classical bounded solution to (2.1) is unique and is given by

$$
u(t, x)=\int_{\mathbb{R}^{N}} G(t, x, y) f(y) d y, \quad \forall(t, x) \in \mathbb{R}_{+} \times \mathbb{R}^{N},
$$

where $G$ is a positive function such that $G(\cdot, \cdot, y) \in C_{l o c}^{1+\delta / 2,2+\delta}\left((0,+\infty) \times \mathbb{R}^{N}\right)$, it solves the equation $D_{t} G(\cdot, \cdot, y)=\mathcal{A} G(\cdot, \cdot, y)$ for almost any $y \in \mathbb{R}^{N}$ and

$$
\int_{\mathbb{R}^{N}} G(t, x, y) d y=1, \quad \forall t>0, x \in \mathbb{R}^{N} .
$$

The function $G$ is called the fundamental solution to problem (2.1). 
The family of bounded operators $\{T(t)\}_{t \geq 0}$ defined by $T(t) f=u(t, \cdot)$ for any $f \in C_{b}\left(\mathbb{R}^{N}\right)$ and any $t>0$, gives rise to a contractive semigroup of linear operators in $C_{b}\left(\mathbb{R}^{N}\right)$. In general, $T(t)$ is neither strongly continuous nor analytic in $C_{b}\left(\mathbb{R}^{N}\right)$. Moreover, the following property holds:

If $\left\{f_{n}\right\}_{n \in \mathbb{N}} \subset C_{b}\left(\mathbb{R}^{N}\right)$ is a bounded sequence such that $\lim _{n \rightarrow+\infty} f_{n}=$

$f \in C_{b}\left(\mathbb{R}^{N}\right)$ uniformly in $B(k)$ for all $k>0$, then $\lim _{n \rightarrow+\infty}\left(T(t) f_{n}\right)(x)=$ $(T(t) f)(x)$ uniformly in $[0, T] \times B(k)$ for all $T, k>0$.

We refer the reader to $[3,8,13$, for the proofs of the previous results.

The solution $u(t, x)=(T(t) f)(x)$ can be approximated with the solutions of Dirichlet problems. To be more precise let $u_{R}$ be the solution to the Dirichlet Cauchy problem

$$
\left\{\begin{array}{lll}
D_{t} u_{R}(t, x)=\mathcal{A} u_{R}(t, x), & t>0, & x \in B(R), \\
u_{R}(t, x)=0, & t>0, & x \in \partial B(R), \\
u_{R}(0, x)=\eta_{R}(x) f(x), & & x \in B(R),
\end{array}\right.
$$

where $f \in C(\bar{B}(R))$, and $\eta_{R}$ is any nonnegative smooth function compactly supported in $B(R)$ and such that $\eta_{R} \equiv 1$ in $B(R / 2)$.

We denote by $\left\{T_{R}(t)\right\}_{t \geq 0}$ the semigroup in $C(\bar{B}(R))$ associated with problem (2.5), so that $u_{R}(t, \cdot)=T_{R}(t)\left(\vartheta_{R} f\right)$; the generator of $T_{R}(t)$ is the operator $A$ : $D\left(A_{R}\right) \rightarrow X$ defined by

$$
D\left(A_{R}\right)=\left\{u \in C(\bar{B}(R)) \bigcap_{1<p<+\infty} W_{l o c}^{2, p}(B(R)): u_{\mid \partial B(R)}=0, \mathcal{A} u \in C(\bar{B}(R))\right\},
$$

and $A_{R} u=\mathcal{A} u$ for any $u \in D\left(A_{R}\right)$; see [11, Corollary 3.1.21].

Now let $f \in C_{b}\left(\mathbb{R}^{N}\right)$ and fix $\varepsilon, T, n>0$. Then we have

$$
\lim _{R \rightarrow \infty}\left\|u-u_{R}\right\|_{C^{1+\delta / 2,2+\delta}([\epsilon, T] \times B(n))}=0 ;
$$

see e.g. [3, Theorem 1.12], [13, Theorem 4.2]. Moreover, the fundamental solution $G_{R}$ to problem (2.5) is positive for any $R>0, G_{R_{1}}<G_{R_{2}}$ for all $R_{1}<R_{2}$, and $G_{R}$ tends to the function $G$ in (2.2) as $R$ tends to $+\infty$. As straightforward consequences, we deduce that, for any $f \geq 0$ and $R_{1}<R_{2}$, we have

$$
T_{R_{1}}(t) f \leq T_{R_{2}}(t) f \leq T(t) f, \quad \forall t>0
$$

(see also [13, Lemma 4.1]), and, for any $f \in C_{b}\left(\mathbb{R}^{N}\right)$,

$$
\left|T(t) f-T_{R}(t) f\right| \leq\left(T(t)-T_{R}(t)\right)|f|, \quad \forall t>0 .
$$

Let us now recall the following classical interior estimate (see e.g. [7] Theorems 3.5 and 3.10]).

Theorem 2.2. Suppose that $q_{i j}, b_{j} \in C_{l o c}^{k+\delta}\left(\mathbb{R}^{N}\right)$ for any $i, j=1, \ldots, N$, for some $k \in \mathbb{N}$ and $\delta \in(0,1)$, and suppose that H1 holds. Let $\Omega \subset \mathbb{R}^{N}$ be a bounded open set and let $u \in L^{\infty}((0, T) \times \Omega)$ be a solution of the equation $u_{t}=\mathcal{A} u$ in $(0, T) \times \Omega$.

Then, for any $|\beta| \leq k$, the function $D^{\beta} u$ belongs to $C_{l o c}^{1+\delta / 2,2+\delta}((0,+\infty) \times$ $\Omega)$. Moreover, for any $\varepsilon, \varepsilon^{\prime}>0\left(\varepsilon<\varepsilon^{\prime}<T\right)$, and any open set $\Omega^{\prime} \subset \subset \Omega$, 
there exists a positive constant $C$ depending on $\varepsilon, \varepsilon^{\prime}, T, \Omega, \Omega^{\prime}, k,\left\|q_{i j}\right\|_{C^{k+\delta}(\Omega)}$ and $\left\|b_{j}\right\|_{C^{k+\delta}(\Omega)}(i, j=1, \ldots, N)$, such that

$$
\|u\|_{C^{1+\delta / 2, k+2+\delta}\left(\left[\varepsilon^{\prime}, T\right] \times \Omega^{\prime}\right)} \leq C \sup _{(t, x) \in[\varepsilon, T] \times \Omega}|u(t, x)| .
$$

Theorem 2.2 yields the following consequence: applying (2.10) to $u-u_{R}$, by (2.7) it follows that

$$
\lim _{R \rightarrow+\infty}\left\|u_{R}-u\right\|_{C^{1+\delta / 2, k+2+\delta}([\epsilon, T] \times B(n))}=0,
$$

for all $f \in C_{b}\left(\mathbb{R}^{N}\right)$ and all fixed $\varepsilon, T, n>0$.

Next, we recall the following result. Even if it is a classical result, to the authors' knowledge it seems not to be explicitly mentioned in the most used classical books; then we give a proof.

Theorem 2.3. Let $R>0$ and let assumption $H 1$ be satisfied. Moreover, assume that the coefficients of $\mathcal{A}$ belong to $C^{k+\alpha}(\bar{B}(R))$ for some $\alpha \in(0,1)$ and $k=1,2,3$. Then for any $f \in C_{0}^{j}(B(R))(j=0, \ldots, k)$ the function $(t, x) \mapsto$ $t^{(k-j) / 2}\left(D^{k} T_{R}(t) f\right)(x)$ is continuous in $[0,+\infty) \times \bar{B}(R)$.

Proof. The proof follows from a density argument. We just sketch it in the case when $j=k=3$. It is well known that for any $f \in C_{0}^{\infty}(B(R))$, the functions $(t, x) \mapsto\left(D^{j} T_{R}(t) f\right)(x)(j=0, \ldots, 3)$ are continuous in $[0,+\infty) \times \bar{B}(R)$ (see e.g. [10, Theorem 5.2]). Using a method similar to the one that we will use in the proof of Theorem 3.3 we can easily show that, for any $T>0$, there exists a positive constant $C=C(R, T)$, such that

$$
\left\|T_{R}(t) f\right\|_{C^{3}(\bar{B}(R))} \leq C\|f\|_{C^{3}(\bar{B}(R))}, \quad \forall t \in[0, T] .
$$

Now, with any $f \in C_{0}^{3}(B(R))$ we associate a sequence of smooth functions $\left\{f_{n}\right\}_{n \in \mathbb{N}}$ $\subset C_{0}^{\infty}(B(R))$ converging to $f$ in $C^{3}(\bar{B}(R))$. Since for any $n \in \mathbb{N}$ the functions $D^{j} T_{R}(\cdot) f_{n}(j=0, \ldots, 3)$ are continuous in $[0,+\infty) \times \bar{B}(R)$, taking (2.12) into account, we easily get the assertion.

We conclude this section with the following version of the maximum principle which will be used throughout all this paper. For the proof, we refer the reader to [12, Proposition 2.1].

Lemma 2.4. Suppose that assumptions $H 1$ and H2 hold true and let $u:[0, T] \times$ $\mathbb{R}^{N} \rightarrow \mathbb{R}$ be a bounded classical solution of the problem

$$
\left\{\begin{array}{lll}
D_{t} u(t, x)=\mathcal{A} u(t, x)+g(t, x), & t \in(0, T), & x \in \mathbb{R}^{N}, \\
u(0, x)=f(x), & x \in \mathbb{R}^{N},
\end{array}\right.
$$

where $f \in C_{b}\left(\mathbb{R}^{N}\right)$ and $g \in C\left((0, T) \times \mathbb{R}^{N}\right)$. If $g \leq 0$, then $u \leq \sup f^{+}$, where $f^{+}(x)=\max \{f(x), 0\}$. Similarly, if $g \geq 0$, then $u \geq \inf f^{-}$, where $f^{-}(x)=$ $\min \{f(x), 0\}$.

\section{UNIFORM ESTIMATES}

In this section, we show that, under suitable assumptions on the coefficients $q_{i j}$ and $b_{j}(i, j=1, \ldots, N)$, for any $\omega>0$, there exist positive constants $C_{k, l}=C_{k, l}(\omega)$ such that

$$
\|T(t) f\|_{C_{b}^{l}\left(\mathbb{R}^{N}\right)} \leq C_{k, l} t^{-(l-k) / 2} e^{\omega t}\|f\|_{C_{b}^{k}\left(\mathbb{R}^{N}\right)}, \quad \forall f \in C_{b}^{k}\left(\mathbb{R}^{N}\right),
$$


for any $k, l \in \mathbb{N}, 0 \leq k \leq l \leq 3$. Once (B.1) is established, one can easily prove, by interpolation, that there exist positive constants $C_{k+\alpha, m+\theta}=C_{k+\alpha, m+\theta}(\omega)$ such that

$\|T(t) f\|_{C_{b}^{m+\theta}\left(\mathbb{R}^{N}\right)} \leq C_{k+\alpha, m+\theta} t^{-(m+\theta-k-\alpha) / 2} e^{\omega t}\|f\|_{C_{b}^{k+\alpha}\left(\mathbb{R}^{N}\right)}, \quad \forall f \in C_{b}^{k+\alpha}\left(\mathbb{R}^{N}\right)$,

for any $m, k=0,1,2, \alpha, \theta \in[0,1], k+\alpha \leq m+\theta$.

Although we limit ourselves to the case when $l \leq 3$, our techniques work as well for $l>3$ under suitable additional assumptions on the coefficients.

We will consider the following assumptions:

H3): there exists a constant $C>0$ such that

$$
\begin{aligned}
\left|\sum_{j=1}^{N} q_{i j}(x) x_{j}\right| \leq & C\left(1+|x|^{2}\right) \nu(x), \quad \sum_{j=1}^{N} q_{j j}(x) \leq C\left(1+|x|^{2}\right) \nu(x), \\
& \sum_{j=1}^{N} b_{j}(x) x_{j} \leq C\left(1+|x|^{2}\right) \nu(x),
\end{aligned}
$$

for any $x \in \mathbb{R}^{N}, i=1, \ldots, N$;

H4-1): $q_{i j}, b_{j} \in C_{l o c}^{1+\delta}\left(\mathbb{R}^{N}\right)$ for some $\delta \in(0,1)$ and there exist a constant $C>0$ and a function $d: \mathbb{R}^{N} \rightarrow \mathbb{R}$ with $L_{1}:=\sup _{x \in \mathbb{R}^{N}}\{d(x) / \nu(x)\}<+\infty$, such that $\left|D_{k} q_{i j}(x)\right| \leq C \nu(x)$ for any $i, j, k=1, \ldots, N$ and

$$
\sum_{i, j=1}^{N} D_{i} b_{j}(x) \xi_{i} \xi_{j} \leq d(x)|\xi|^{2}, \quad \forall x, \xi \in \mathbb{R}^{N} ;
$$

H4-2): $q_{i j}, b_{j} \in C_{l o c}^{2+\delta}\left(\mathbb{R}^{N}\right)$ for some $\delta \in(0,1)$, hypothesis $H_{4}$ - 1 holds true and there exist a positive function $r: \mathbb{R}^{N} \rightarrow \mathbb{R}$ and three constants $K_{1} \in \mathbb{R}$, $L_{2}, L_{3}>0$ such that $\left|D^{\beta} b_{j}(x)\right| \leq r(x)$ for any $j=1, \ldots, N$ and any $|\beta|=2$, $d(x)+L_{2} r(x) \leq L_{3} \nu(x)$ and

$$
\sum_{i, j, h, k=1}^{N} D_{h k} q_{i j}(x) m_{i j} m_{h k} \leq K_{1} \nu(x) \sum_{h, k=1}^{N} m_{h k}^{2},
$$

for any symmetric matrix $M=\left(m_{h k}\right)_{h, k=1}^{N}$ and any $x \in \mathbb{R}^{N}$;

H4-3): $q_{i j}, b_{j} \in C_{l o c}^{3+\delta}\left(\mathbb{R}^{N}\right)$ for some $\delta \in(0,1)$, hypothesis $H_{4}$-2 holds true and there exists a constant $C>0$ such that $\left|D^{\beta} b_{j}(x)\right| \leq r(x)$ and $\left|D^{\beta} q_{i j}(x)\right| \leq$ $C \nu(x)$ for any $i, j=1, \ldots, N$, any $|\beta|=3$ and any $x \in \mathbb{R}^{N}$.

Remark 3.1. We remark that in $H_{4}-l(l=2,3)$ we can take $r(x)=L_{4}(1+|d(x)|)$ for some $L_{4}>0$. It is sufficient to take $L_{2}<L_{4}^{-1}$ and

$$
L_{3}=\left(1+L_{2} L_{4}\right) \sup _{x \in \mathbb{R}^{N}}(d(x) / \nu(x))+L_{2} L_{4} \nu_{0}^{-1}
$$

in $\mathrm{H}_{4}$ - 2 .

Remark 3.2. In some situation, condition $H 3$ is easily implied by $H_{4}-1$. This is the case, for instance, when there exists a positive constant $K$ such that

$$
\int_{0}^{1} \nu(t x) d t \leq K \nu(x), \quad \forall x \in \mathbb{R}^{N}
$$


We limit ourselves to showing that in such a situation, condition (3.4) follows from H4-1. The same argument can be used to prove that also (3.3) is a consequence of H4- 1 .

To show (3.4) we observe that taking $(x, \xi)=(t z, z)$ in $H_{4}$ - 1 gives

$$
\frac{d}{d t} \sum_{j=1}^{N} b_{j}(t z) z_{j} \leq L_{1} \nu(t z)|z|^{2}, \quad \forall z \in \mathbb{R}^{N}
$$

Integrating (3.6) with respect to $t \in[0,1]$ and taking 3.5 into account, we get

$$
\begin{aligned}
\sum_{j=1}^{N} b_{j}(z) z_{j} & \leq \sum_{j=1}^{N} b_{j}(0) z_{j}+L_{1}|z|^{2} \int_{0}^{1} \nu(t z) d t \\
& \leq|z||b(0)|+K L_{1}|z|^{2} \nu(z) \\
& \leq C^{\prime}|z|(1+|z|) \nu(z),
\end{aligned}
$$

for any $z \in \mathbb{R}^{N}$, where $|b(0)|$ denotes the euclidean norm of the vector $b(0)=$ $\left(b_{1}(0), \ldots, b_{N}(0)\right)$ and $C^{\prime}=\max \left\{|b(0)| \nu_{0}^{-1}, K L_{1}\right\}$.

Let us observe that (3.5) is satisfied, for instance, in the case when $\nu(x)=\bar{\nu}(|x|)$ for any $x \in \mathbb{R}^{N}$ and some nondecreasing function $\bar{\nu}: \mathbb{R}_{+} \rightarrow \mathbb{R}_{+}$, and in the case when $\nu(x)=c+\widetilde{\nu}(x), c$ and $\widetilde{\nu}$ being, respectively, a positive constant and a homogeneous function of degree $\alpha>0$.

Theorem 3.3. Let $l \in\{1,2,3\}$, and let assumptions H1-H3, H4-l be satisfied. Then, for any $\omega>0$ and any $k=0, \ldots, l$, there exist constants $C_{k, l}=C_{k, l}(\omega)>0$ such that (3.1) holds true. In particular, if $k=l$ we can take $\omega=0$ in (3.1).

Proof. We begin the proof by considering the case when $k=0$ and $l=3$. Let $\varphi \in$ $C^{\infty}([0,+\infty))$ be a nonincreasing function such that $\varphi(t)=1$ for any $t \in[0,1 / 2)$, and $\varphi(t)=0$ for any $t \in[3 / 4,+\infty)$. With any $R>0$ we associate the function $\eta=\eta_{R}: \mathbb{R}^{N} \rightarrow \mathbb{R}$ defined by $\eta(x)=\varphi(|x| / R)$. Of course $\eta \in C_{0}^{\infty}\left(\mathbb{R}^{N}\right), \eta=1$ in $B(R / 2)$ and $\eta=0$ outside the ball $B(R)$.

We fix $T>0$ and define the function

$$
\begin{aligned}
v_{0,3, R}(t, x)= & \left|u_{R}(t, x)\right|^{2}+a t \eta^{2}\left|D u_{R}(t, x)\right|^{2}+a^{2} t^{2} \eta^{4}\left|D^{2} u_{R}(t, x)\right|^{2} \\
& +a^{3} t^{3} \eta^{6}\left|D^{3} u_{R}(t, x)\right|^{2}
\end{aligned}
$$

for any $t \in[0, T]$ and any $x \in B(R)$, where $u_{R}(t, x)=\left(T_{R}(t)(\eta f)\right)(x)$ is the solution of the Dirichlet Cauchy problem (2.5). Taking Theorem 2.3 into account, it follows that $v_{0,3, R}$ is continuous in $[0, T] \times \bar{B}(R)$, and, with some computations, one can see that $v_{0,3, R}$ solves the Cauchy problem

$$
\left\{\begin{array}{lll}
D_{t} v_{0,3, R}(t, x)=\mathcal{A} v_{0,3, R}(t, x)+g_{R}(t, x), & t \in[0, T], & x \in B(R), \\
v_{0,3, R}(t, x)=0, & t \in[0, T], & x \in \partial B(R), \\
v_{0,3, R}(0, x)=(\eta f)^{2}(x), & & x \in \bar{B}(R),
\end{array}\right.
$$


where $g_{R}(t, x)=\sum_{j=1}^{8} g_{j, R}(t, x)$ for any $t>0$ and any $x \in B(R)$, with

$$
\begin{aligned}
g_{1, R}= & -2 \sum_{i, j=1}^{N} q_{i j} D_{i} u_{R} D_{j} u_{R}-2 a t \eta^{2} \sum_{i, j, h=1}^{N} q_{i j} D_{i h} u_{R} D_{j h} u_{R} \\
& -2 a^{2} t^{2} \eta^{4} \sum_{i, j, h, k=1}^{N} q_{i j} D_{i h k} u_{R} D_{j h k} u_{R} \\
& -2 a^{3} t^{3} \eta^{6} \sum_{i, j, h, k, l=1}^{N} q_{i j} D_{i h k l} u_{R} D_{j h k l} u_{R},
\end{aligned}
$$

$$
g_{2, R}=-2\left(a t\left|D u_{R}\right|^{2}+6 a^{2} t^{2} \eta^{2}\left|D^{2} u_{R}\right|^{2}+15 a^{3} t^{3} \eta^{4}\left|D^{3} u_{R}\right|^{2}\right) \sum_{i, j=1}^{N} q_{i j} D_{i} \eta D_{j} \eta,
$$

$$
\begin{aligned}
g_{3, R}= & -2\left(a t \eta\left|D u_{R}\right|^{2}+2 a^{2} t^{2} \eta^{3}\left|D^{2} u_{R}\right|^{2}+3 a^{3} t^{3} \eta^{5}\left|D^{3} u_{R}\right|^{2}\right) \sum_{i, j=1}^{N} q_{i j} D_{i j} \eta \\
& -2\left(a t \eta\left|D u_{R}\right|^{2}+2 a^{2} t^{2} \eta^{3}\left|D^{2} u_{R}\right|^{2}+3 a^{3} t^{3} \eta^{5}\left|D^{3} u_{R}\right|^{2}\right) \sum_{j=1}^{N} b_{j} D_{j} \eta \\
& -8 a t \eta \sum_{i, j, h=1}^{N} q_{i j} D_{j} \eta D_{h} u_{R} D_{i h} u_{R}-16 a^{2} t^{2} \eta^{3} \sum_{i, j, h, k=1}^{N} q_{i j} D_{j} \eta D_{h k} u_{R} D_{i h k} u_{R} \\
& -24 a^{3} t^{3} \eta^{5} \sum_{i, j, h, k, l=1}^{N} q_{i j} D_{j} \eta D_{h k l} u_{R} D_{i h k l} u_{R}, \\
g_{4, R}= & 2 a t \eta^{2} \sum_{j, h=1}^{N} D_{h} b_{j} D_{j} u_{R} D_{h} u_{R}+4 a^{2} t^{2} \eta^{4} \sum_{j, h, k=1}^{N} D_{h} b_{j} D_{j k} u_{R} D_{h k} u_{R} \\
& +6 a^{3} t^{3} \eta^{6} \sum_{j, h, k, l=1}^{N} D_{h} b_{j} D_{j k l} u_{R} D_{h k l} u_{R},
\end{aligned}
$$

$$
\begin{aligned}
g_{5, R}= & 2 a t \eta^{2} \sum_{i, j, h=1}^{N} D_{h} q_{i j} D_{h} u_{R} D_{i j} u_{R}+4 a^{2} t^{2} \eta^{4} \sum_{i, j, h, k=1}^{N} D_{h} q_{i j} D_{h k} u_{R} D_{i j k} u_{R} \\
& +6 a^{3} t^{3} \eta^{6} \sum_{i, j, h, k, l=1}^{N} D_{h} q_{i j} D_{h k l} u_{R} D_{i j k l} u_{R}
\end{aligned}
$$




$$
\begin{aligned}
g_{6, R}= & 2 a^{2} t^{2} \eta^{4} \sum_{i, j, h, k=1}^{N} D_{h k} q_{i j} D_{i j} u_{R} D_{h k} u_{R} \\
& +6 a^{3} t^{3} \eta^{6} \sum_{i, j, h, k, l=1}^{N} D_{h k} q_{i j} D_{i j l} u_{R} D_{h k l} u_{R} \\
& +2 a^{2} t^{2} \eta^{4} \sum_{j, h, k=1}^{N} D_{h k} b_{j} D_{j} u_{R} D_{h k} u_{R} \\
& +6 a^{3} t^{3} \eta^{6} \sum_{j, h, k, l=1}^{N} D_{h k} b_{j} D_{j l} u_{R} D_{h k l} u_{R},
\end{aligned}
$$

$$
\begin{gathered}
g_{7, R}=2 a^{3} t^{3} \eta^{6}\left(\sum_{i, j, h, k, l=1}^{N} D_{h k l} q_{i j} D_{i j} u_{R} D_{h k l} u_{R}+\sum_{j, h, k, l=1}^{N} D_{h k l} b_{j} D_{j} u_{R} D_{h k l} u_{R}\right), \\
(3.16) \quad g_{8, R}=a \eta^{2}\left|D u_{R}\right|^{2}+2 a^{2} t \eta^{4}\left|D^{2} u_{R}\right|^{2}+3 a^{3} t^{2} \eta^{6}\left|D^{3} u_{R}\right|^{2} .
\end{gathered}
$$

Taking the ellipticity condition $H 1$ into account, we easily deduce that

$$
g_{1, R} \leq-2 \nu\left|D u_{R}\right|^{2}-2 a t \eta^{2} \nu\left|D^{2} u_{R}\right|^{2}-2 a^{2} t^{2} \eta^{4} \nu\left|D^{3} u_{R}\right|^{2}-2 a^{3} t^{3} \eta^{6} \nu\left|D^{4} u_{R}\right|^{2}
$$

and that $g_{2, R} \leq 0$.

To estimate the function $g_{3, R}$ we observe that, by virtue of (3.3), and recalling that $\varphi$ is nonincreasing in $[0,+\infty)$ and $\varphi^{\prime}=0$ in $[0,1 / 2)$, it can be easily shown that

$$
\begin{aligned}
& \left|\sum_{i, j=1}^{N} q_{i j}(x) D_{i j} \eta(x)\right| \\
\leq & \left|\varphi^{\prime}(|x| / R)\right|\left(\frac{1}{|x| R} \sum_{j=1}^{N} q_{j j}(x)+\frac{1}{|x|^{3} R} \sum_{i, j=1}^{N} q_{i j}(x) x_{i} x_{j}\right) \\
& +\left|\varphi^{\prime \prime}(|x| / R)\right| \frac{1}{|x|^{2} R^{2}} \sum_{i, j=1}^{N} q_{i j}(x) x_{i} x_{j} \\
\leq & C \nu(x) \sup _{|x| \leq R}\left[\left|\varphi^{\prime}(|x| / R)\right|\left(\frac{1+|x|^{2}}{|x| R}+\frac{1+|x|^{2}}{|x|^{3} R} \sum_{i=1}^{N}\left|x_{i}\right|\right)\right. \\
& \left.+C\left|\varphi^{\prime \prime}(|x| / R)\right| \frac{1+|x|^{2}}{|x|^{2} R^{2}} \sum_{i=1}^{N}\left|x_{i}\right|\right] \\
\leq & 4 C(1+2 \sqrt{N})\left\|\varphi^{\prime}\right\|_{\infty} \nu(x)+4 C \sqrt{N}\left\|\varphi^{\prime \prime}\right\|_{\infty} \nu(x) \\
= & : C^{\prime} \nu(x),
\end{aligned}
$$


for any $x \in B(R)$ and any $R \geq 1$. Similarly,

$$
\begin{aligned}
\left|\sum_{j=1}^{N} q_{i j}(x) D_{j} \eta(x)\right| & \leq C \nu(x) \sup _{|x| \leq R}\left(\left|\varphi^{\prime}(|x| / R)\right| \frac{1+|x|^{2}}{|x| R}\right) \\
& \leq 4 C\left\|\varphi^{\prime}\right\|_{\infty} \nu(x)=: C^{\prime \prime} \nu(x) .
\end{aligned}
$$

Using (3.4) and recalling that $\varphi$ is nonincreasing in $[0,+\infty)$, we can easily show that

$$
\begin{aligned}
-\sum_{j=1}^{N} b_{j}(x) D_{j} \eta(x) & =-\varphi^{\prime}(|x| / R) \frac{1}{|x| R} \sum_{j=1}^{N} b_{j}(x) x_{j} \\
& \leq C \nu(x) \sup _{|x| \leq R}\left(-\varphi^{\prime}(|x| / R) \frac{1+|x|^{2}}{|x| R}\right) \\
& \leq C^{\prime \prime} \nu(x),
\end{aligned}
$$

for any $x \in B(R)$.

Taking (3.18), (3.19) and (3.20) into account and recalling that for any $a, b, \varepsilon>0$ it holds that $a b \leq(4 \varepsilon)^{-1} a^{2}+\varepsilon b^{2}$, we can now easily show that

$$
\begin{aligned}
g_{3, R} \leq & 2 a C^{\prime} t \nu \eta\left|D u_{R}\right|^{2}+4 a^{2} C^{\prime} t^{2} \nu \eta^{3}\left|D^{2} u_{R}\right|^{2}+6 a^{3} C^{\prime} t^{3} \nu \eta^{5}\left|D^{3} u_{R}\right|^{2} \\
& +2 a C^{\prime \prime} t \nu \eta\left|D u_{R}\right|^{2}+4 a^{2} C^{\prime \prime} t^{2} \nu \eta^{3}\left|D^{2} u_{R}\right|^{2}+6 a^{3} C^{\prime \prime} t^{3} \nu \eta^{5}\left|D^{3} u_{R}\right|^{2} \\
& +8 a C^{\prime \prime} t \nu\left(\frac{N}{4 \varepsilon}\left|D u_{R}\right|^{2}+\varepsilon \eta^{2}\left|D^{2} u_{R}\right|^{2}\right) \\
& +16 a^{2} C^{\prime \prime} t^{2} \nu\left(\frac{N}{4 \varepsilon} \eta^{2}\left|D^{2} u_{R}\right|^{2}+\varepsilon \eta^{4}\left|D^{3} u_{R}\right|^{2}\right) \\
& +24 a^{3} C^{\prime \prime} t^{3} \nu\left(\frac{N}{4 \varepsilon} \eta^{4}\left|D^{3} u_{R}\right|^{2}+\varepsilon \eta^{6}\left|D^{4} u_{R}\right|^{2}\right) \\
\leq & 2 a\left(C^{\prime}+C^{\prime \prime}+C^{\prime \prime} \frac{N}{\varepsilon}\right) t \nu\left|D u_{R}\right|^{2} \\
& +4 a\left(a C^{\prime} t+a C^{\prime \prime} t+2 C^{\prime \prime} \varepsilon+a C^{\prime \prime} t \frac{N}{\varepsilon}\right) t \nu \eta^{2}\left|D^{2} u_{R}\right|^{2} \\
& +2 a^{2}\left(3 a C^{\prime} t+3 a C^{\prime \prime} t+8 C^{\prime \prime} \varepsilon+3 a C^{\prime \prime} t \frac{N}{\varepsilon}\right) t^{2} \nu \eta^{4}\left|D^{3} u_{R}\right|^{2} \\
& +24 a^{3} C^{\prime \prime} t^{3} \varepsilon \nu \eta^{6}\left|D^{4} u_{R}\right|^{2} .
\end{aligned}
$$

Taking advantage of assumption $\mathrm{H}_{4}$-1, we deduce that

$$
g_{4, R} \leq 2 a t d \eta^{2}\left|D u_{R}\right|^{2}+4 a^{2} t^{2} d \eta^{4}\left|D^{2} u_{R}\right|^{2}+6 a^{3} t^{3} d \eta^{6}\left|D^{3} u_{R}\right|^{2} .
$$


The terms $g_{5, R}, g_{6, R}$ and $g_{7, R}$ can be estimated in a similar way by taking assumptions $\mathrm{H}_{4}$ - 2 and $\mathrm{H}_{4}$-3 into account, and they yield

$$
\begin{aligned}
g_{5, R} \leq & a t C \frac{N^{2}}{2 \varepsilon} \nu\left|D u_{R}\right|^{2}+a C N\left(2 \varepsilon+a t \frac{N}{\varepsilon}\right) t \nu \eta^{2}\left|D^{2} u_{R}\right|^{2} \\
& +a^{2} C N\left(4 \varepsilon+3 a t \frac{N}{2 \varepsilon}\right) t^{2} \nu \eta^{4}\left|D^{3} u_{R}\right|^{2}+6 a^{3} t^{3} \varepsilon C N \nu \eta^{4}\left|D^{4} u_{R}\right|^{2},
\end{aligned}
$$

$$
\begin{aligned}
& g_{6, R} \leq a^{2} t^{2} \eta^{2} r \frac{N^{2}}{2 \varepsilon}\left|D u_{R}\right|^{2}+a^{2} t^{2} \eta^{4}\left[2 K_{1} \nu+N\left(2 \varepsilon+3 a t \frac{N}{2 \varepsilon}\right) r\right]\left|D^{2} u_{R}\right|^{2} \\
&+6 a^{3} t^{3} \eta^{6}\left(K_{1} \nu+\varepsilon N r\right)\left|D^{3} u_{R}\right|^{2} \\
& g_{7, R} \leq a^{3} t^{3} \eta^{2} r \frac{N^{3}}{2 \varepsilon}\left|D u_{R}\right|^{2}+a^{3} t^{3} \eta^{4} C \nu \frac{N^{3}}{2 \varepsilon}\left|D^{2} u_{R}\right|^{2} \\
&+2 a^{3} t^{3} \eta^{6} \varepsilon N(C \nu N+r)\left|D^{3} u_{R}\right|^{2}
\end{aligned}
$$

From (3.17), (3.21)-(3.25) we immediately deduce that for any $t \in[0, T]$ we have $(3.26)$

$$
\begin{aligned}
& g_{R} \leq\left\{-\nu_{0}+a+\nu\left[-1+2 a t\left(C^{\prime}+C^{\prime \prime}+C^{\prime \prime} \frac{N}{\varepsilon}\right)+a t C \frac{N^{2}}{2 \varepsilon}+a^{2} t^{2} C \frac{N^{2}}{2 \varepsilon}\right]\right. \\
& \left.+a t\left[2 d+a t(1+a t N) \frac{N^{2}}{2 \varepsilon} r\right]\right\}\left|D u_{R}\right|^{2} \\
& +a\left\{-\nu_{0}+2 a+\nu\left[-1+4\left(a C^{\prime} t+a C^{\prime \prime} t+2 C^{\prime \prime} \varepsilon+a C^{\prime \prime} t \frac{N}{\varepsilon}\right)\right.\right. \\
& \left.+C N\left(2 \varepsilon+a t \frac{N}{\varepsilon}\right)+2 K_{1}^{+} a t+a^{2} t^{2} C \frac{N^{3}}{2 \varepsilon}\right] \\
& \left.+a t\left[4 d+\left(2 \varepsilon N+3 a t \frac{N^{2}}{2 \varepsilon}\right) r\right]\right\} t \eta^{2}\left|D^{2} u_{R}\right|^{2} \\
& +a^{2}\left\{-\nu_{0}+3 a+\nu\left[-1+2\left(3 a C^{\prime} t+3 a C^{\prime \prime} t+8 C^{\prime \prime} \varepsilon+3 a C^{\prime \prime} t \frac{N}{\varepsilon}\right)\right.\right. \\
& \left.+C N\left(4 \varepsilon+3 a t \frac{N}{2 \varepsilon}\right)+6 a t K_{1}^{+}+2 a t \varepsilon C N^{2}\right] \\
& +2 a t(3 d+4 \varepsilon N r)\} t^{2} \eta^{4}\left|D^{3} u_{R}\right|^{2} \\
& +2 a^{3}\left(-1+12 C^{\prime \prime} \varepsilon+3 \varepsilon C N\right) t^{3} \nu \eta^{6}\left|D^{4} u_{R}\right|^{2} .
\end{aligned}
$$


We now choose $(a, \varepsilon)$, sufficiently small, satisfying the following set of inequalities:

$$
\left\{\begin{array}{l}
3 a-\nu_{0} \leq 0, \quad 4 \varepsilon N \leq 3 L_{2}, \\
a T(1+a T N) \frac{N^{2}}{2 \varepsilon} \leq 2 L_{2}, \quad 2 \varepsilon N+3 a T \frac{N^{2}}{2 \varepsilon} \leq 4 L_{2}, \\
-1+a T\left[4\left(C^{\prime}+C^{\prime \prime}+C^{\prime \prime} \frac{N}{\varepsilon}+L_{3}\right)+C \frac{N^{2}}{\varepsilon}+2 K_{1}^{+}\right] \\
\quad+2 \varepsilon\left(C N+4 C^{\prime \prime}\right)+a^{2} T^{2} C \frac{N^{3}}{2 \varepsilon} \leq 0, \\
-1+a T\left[6\left(C^{\prime}+C^{\prime \prime}+C^{\prime \prime} \frac{N}{\varepsilon}+K_{1}^{+}+L_{3}\right)+3 C \frac{N^{2}}{2 \varepsilon}+2 \varepsilon C N^{2}\right] \\
\quad+4 \varepsilon\left(C N+4 C^{\prime \prime}\right) \leq 0 .
\end{array}\right.
$$

With such a choice of $(a, \varepsilon)$ we get $g_{R}(t, x) \leq 0$ for any $t \in[0, T]$ and any $x \in B(R)$. From the maximum principle we now deduce that

$$
\left|v_{0,3, R}(t, x)\right| \leq\|\eta f\|_{\infty}^{2} \leq\|f\|_{\infty}^{2}, \quad \forall t \in[0, T], x \in B(R) .
$$

By (2.11), taking the limit as $R$ tends to $+\infty$, we deduce that (3.1) holds for $t \in[0, T]$, for some constant $C_{0,3}=C_{0,3}(T)>0$ and with $\omega=0$. Using the semigroup property we can then extend the estimate to all the positive $t$. Indeed, for any $\omega>0$ we can choose $T=T(\omega)$ such that $e^{\omega t} t^{-3 / 2} \geq 1$ for all $t>T(\omega)$, and then if $t>T$ we get

$$
\begin{aligned}
\|T(t) f\|_{C_{b}^{3}\left(\mathbb{R}^{N}\right)} & =\|T(T) T(t-T) f\|_{C_{b}^{3}\left(\mathbb{R}^{N}\right)} \leq \frac{C_{0,3}}{T^{3 / 2}}\|T(t-T) f\|_{\infty} \\
& \leq \frac{C_{0,3}}{T^{3 / 2}}\|f\|_{\infty} \leq \frac{C_{0,3}}{T^{3 / 2}} \frac{e^{\omega t}}{t^{3 / 2}}\|f\|_{\infty},
\end{aligned}
$$

and therefore (3.1) follows with $C_{0,3}(\omega)=\max \left\{C_{0,3}(T), C_{0,3}(T) T^{-3 / 2}\right\}$.

In the other cases the proof is very similar. It suffices to apply the arguments above to the function

$$
v_{k, l, R}(t, x)=\sum_{j=0}^{l} a^{j} t^{(j-k)^{+}}(\eta(x))^{2 j}\left|D^{j} u_{R}(t, x)\right|^{2}, \quad \forall t>0, x \in B(R), \quad k \leq l \leq 3 .
$$

Let us just show that, if $k=l$, we can take $\omega=0$ in (3.1). We only consider the case when $l=3$. A straightforward computation shows that $v_{3,3, R}$ is a classical solution to the Dirichlet Cauchy problem (3.8) with $v_{3,3, R}(0, \cdot)=\sum_{j=0}^{3}\left|D^{j}(\eta f)\right|^{2}$ and $g_{R}$ being replaced by $\widetilde{g}_{R}=\sum_{j=1}^{7} \widetilde{g}_{j, R}$, where $\widetilde{g}_{j, R}(j=1, \ldots, 7)$ are defined by the right-hand sides of (3.9)- 3.15 after replacing each $t$, therein explicitly appearing, with $t=1$. Arguing as above we can easily show that $\widetilde{g}_{R}$ can be estimated for any $t>0$ by the last side of (3.26), where we set $T=1$ and replace the terms $-\nu_{0}+a,-\nu_{0}+2 a$ and $-\nu_{0}+3 a$ simply by $-\nu$. It is now clear that we can take $(a, \varepsilon)$ such that $g_{R}(t, x) \leq 0$ for any $t>0$ and any $x \in \mathbb{R}^{N}$, and consequently

$$
\left|v_{3,3, R}(t, x)\right| \leq\|\eta f\|_{\infty}^{2}+\|D(\eta f)\|_{\infty}^{2}+\left\|D^{2}(\eta f)\right\|_{\infty}^{2}+\left\|D^{3}(\eta f)\right\|_{\infty}^{2},
$$

for any $t \geq 0$ and any $x \in \mathbb{R}^{N}$, which yields (3.1) taking the limit as $R$ tends to $+\infty$.

Now, by interpolation, we can prove (3.2). 
Theorem 3.4. Let $l \in\{1,2,3\}$ and let assumptions H1-H3, H4-l be satisfied. Then, for any $\omega>0$, any $k, m=0, \ldots, l-1$ and any $\alpha, \theta \in[0,1]$ such that $k+\alpha \leq m+\theta$, there exist constants $C_{k+\alpha, m+\theta}=C_{k+\alpha, m+\theta}(\omega)>0$ such that (3.2) holds true. In particular, if $k+\alpha=m+\theta$ we can take $\omega=0$ in (3.2).

Proof. The proof follows easily from an interpolation argument. We limit ourselves to sketching it in a particular case, since the same techniques can also be applied to all the other cases. So, let us assume that $l=3, k=m=2$ and $0<\alpha \leq \theta<1$, and fix $\omega>0$. From (3.1) with $(k, l)=(2,2)$ and $(k, l)=(2,3)$, we deduce that

$$
\|T(t)\|_{L\left(C_{b}^{2}\left(\mathbb{R}^{N}\right), C_{b}^{2}\left(\mathbb{R}^{N}\right)\right)} \leq C_{2,2}, \quad\|T(t)\|_{L\left(C_{b}^{2}\left(\mathbb{R}^{N}\right), C_{b}^{3}\left(\mathbb{R}^{N}\right)\right)} \leq C_{2,3} t^{-\frac{1}{2}} e^{\omega t},
$$

for any $t>0$. Recalling that $\left(C_{b}^{2}\left(\mathbb{R}^{N}\right) ; C_{b}^{3}\left(\mathbb{R}^{N}\right)\right)_{\beta, \infty}=C_{b}^{2+\beta}\left(\mathbb{R}^{N}\right)$, for any $\beta \in(0,1)$ (see [11 Theorem 1.2.17]), and applying a well-known result in interpolation (see [11, Proposition 1.2.6]), we easily see from (3.27) that $T(t)$ is bounded from $C_{b}^{2}\left(\mathbb{R}^{N}\right)$ into $C_{b}^{2+\beta}\left(\mathbb{R}^{N}\right)$ for any $\beta \in(0,1)$ and

$$
\|T(t)\|_{L\left(C_{b}^{2}\left(\mathbb{R}^{N}\right), C_{b}^{2+\beta}\left(\mathbb{R}^{N}\right)\right)} \leq C_{2,2+\beta} t^{-\beta / 2} e^{\beta \omega t}, \quad \forall t>0,
$$

where $C_{2,2+\beta}=C_{2,2}^{1-\beta} C_{2,3}^{\beta}$.

Applying the same argument to (3.1), with $(k, l)=(3,3)$ and to (3.28), we deduce that $T(t)$ is bounded from $C_{b}^{2+\alpha}\left(\mathbb{R}^{N}\right)$ into $C_{b}^{2+\beta+(1-\beta) \alpha}\left(\mathbb{R}^{N}\right)$ and

$$
\|T(t)\|_{L\left(C_{b}^{2+\alpha}\left(\mathbb{R}^{N}\right), C_{b}^{2+\beta+(1-\beta) \alpha}\left(\mathbb{R}^{N}\right)\right)} \leq C_{2+\alpha, 2+\beta+(1-\beta) \alpha} t^{-(1-\alpha) \beta / 2} e^{(1-\alpha) \beta \omega t},
$$

for any $t>0$, where $C_{2+\alpha, 2+\beta+(1-\beta) \alpha}=C_{2,2+\beta}^{1-\alpha} C_{3,3}^{\alpha}$. Now the assertion follows, taking $\beta=(\theta-\alpha) /(1-\alpha)$.

In some cases, we can extend Theorems 3.3 and 3.4 to the case when in $H_{4}-j$, the $j$-th-order derivatives of the coefficients are merely continuous in $\mathbb{R}^{N}$. As the following theorem shows, this is the case when condition (3.5) is satisfied and there exist $m, M>0$ such that

$$
\int_{0}^{1} d(t x+y) d t \leq M \nu(x+y), \quad \forall x \in \mathbb{R}^{N}, y \in B(m) .
$$

As is immediately seen, the previous condition is satisfied, for instance, when $d$ is bounded from above.

Theorem 3.5. Suppose that assumptions H1-H3, $\mathrm{H}_{4}-\mathrm{j}$ (with the $\mathrm{j}$-th-order derivatives of the coefficients merely continuous in $\mathbb{R}^{N}$ ) and conditions (3.5) and (3.29) are satisfied. Then, (3.1) and (3.2) hold true for any $k, l \in \mathbb{N}, k \leq l \leq j$.

Proof. The proof follows from a density argument. Of course, we can limit ourselves to dealing with (3.1), since, as Theorem 3.4 shows, (3.2) follows easily from (3.1).

For any $\varepsilon>0$, let $\varphi^{\varepsilon}(x)=\varepsilon^{-N} \varphi(x / \varepsilon)$, where $\varphi \in C_{0}^{\infty}\left(\mathbb{R}^{N}\right)$ is any nonnegative even function compactly supported in $B(1)$ with integral 1 . We denote by $f^{\varepsilon}$ the convolution between $f$ and $\varphi^{\varepsilon}$.

Let $\mathcal{A}^{\varepsilon}$ be defined as $\mathcal{A}$ with $q_{i j}$ and $b_{j}$ being replaced, respectively, by $q_{i j}^{\varepsilon}$ and $b_{j}^{\varepsilon}$. As it is immediately seen, $q_{i j}^{\varepsilon}$ and $b_{j}^{\varepsilon}(i, j=1, \ldots, N)$ satisfy assumptions $H 1, H_{4}-j$, with $\nu(x), d(x), r(x)$ being replaced by $\nu^{\varepsilon}(x), d^{\varepsilon}(x)$ and $r^{\varepsilon}(x), C^{\varepsilon}=C, L_{i}^{\varepsilon}=L_{i}$ for $i=1,2,3, K_{1}^{\varepsilon}=K_{1}$ (if $j>1$ ) and $\nu_{0}^{\varepsilon} \geq \nu_{0}$. 
Let us check that $q_{i j}^{\varepsilon}$ and $b_{j}^{\varepsilon}(i, j=1, \ldots, N)$ satisfy condition $H 3$ for some positive constant independent of $\varepsilon$. For this purpose, we observe that (3.5) implies that

$$
\left|q_{i j}(x)\right| \leq\left|q_{i j}(0)\right|+C K \sqrt{N}|x| \nu(x), \quad \forall x \in \mathbb{R}^{N}, i, j=1, \ldots, N,
$$

which yields 3.3.

A straightforward computation now shows that

$$
\left|q_{i j}^{\varepsilon}(x)\right| \leq\left|q_{i j}(0)\right|+C K \sqrt{N}(|x|+\varepsilon) \nu^{\varepsilon}(x), \quad \forall x \in \mathbb{R}^{N}, i, j=1, \ldots, N,
$$

so that the $q_{i j}^{\varepsilon}$ 's satisfy condition (3.3) with a constant independent of $\varepsilon \in(0,1]$.

Similarly, we can show that the $b_{j}^{\varepsilon}$ 's satisfy (3.4) with a positive constant independent of $\varepsilon \leq m$. Indeed, combining (3.7) and (3.29) we deduce that

$$
\begin{aligned}
\int_{0}^{1} d^{\varepsilon}(t x) d t & =\int_{B(\varepsilon)} d y \int_{0}^{1} d(t x-y) \varphi^{\varepsilon}(y) d t \\
& \leq M \int_{B(\varepsilon)} d y \int_{0}^{1} \nu(x-y) \varphi^{\varepsilon}(y) d t \\
& =M \nu^{\varepsilon}(x),
\end{aligned}
$$

for any $x \in \mathbb{R}^{N}$ and $\varepsilon \leq m$. Now, arguing as in the proof of (3.7) we get

$$
\sum_{j=1}^{N} b_{j}^{\varepsilon}(x) x_{j} \leq \sum_{j=1}^{N} b_{j}^{\varepsilon}(0) x_{j}+|x|^{2} \int_{0}^{1} d^{\varepsilon}(t x) d t \leq \sup _{y \in B(\varepsilon)}|b(y)||x|+M|x|^{2} \nu^{\varepsilon}(x),
$$

for any $x \in \mathbb{R}^{N}$, which yields (3.4) with a constant, independent of $\varepsilon \leq m$.

Now, applying the same arguments as in the proof of Theorem 3.3 we can show that for any $\omega>0$ and any $k, l \in \mathbb{N}, 0 \leq k \leq l \leq j$, there exists a positive constant $C_{k, l}=C_{k, l}(\omega)$, independent of $\varepsilon, R$, such that

$$
\left\|T_{R}^{\varepsilon}(t) f\right\|_{C^{l}(\bar{B}(R))} \leq C_{k, l} \frac{e^{\omega t}}{t^{(l-k) / 2}}\|f\|_{C^{k}(\bar{B}(R))}, \quad \forall t>0, \quad f \in C_{b}^{k}(\bar{B}(R)),
$$

where $T_{R}^{\varepsilon}$ is defined as $T_{R}$ with $\mathcal{A}$ being replaced by $\mathcal{A}^{\varepsilon}$. As $R$ tends to $+\infty$, $T_{R}^{\varepsilon}(t) f$ tends to a solution $u^{\varepsilon}=: T^{\varepsilon}(t) f$ to the Cauchy problem (1.1) (with $\mathcal{A}$ being replaced by $\mathcal{A}^{\varepsilon}$ ) which satisfies

$$
\left\|T^{\varepsilon}(t) f\right\|_{C_{b}^{l}\left(\mathbb{R}^{N}\right)} \leq C_{k, l} \frac{e^{\omega t}}{t^{(l-k) / 2}}\|f\|_{C_{b}^{k}\left(\mathbb{R}^{N}\right)}, \quad \forall t>0
$$

(see Section 2). Theorem 2.2 and (3.30) easily imply that there exists an infinitesimal sequence $\left\{\varepsilon_{n}\right\}_{n \in \mathbb{N}}$ such that $T^{\varepsilon_{n}}(t) f$ and its space derivatives up to the $(j-1)$-th-order converge in $C_{l o c}^{1+\delta / 2,2+\delta}\left((0,+\infty) \times \mathbb{R}^{N}\right)$ to a function $u=: S(t) f$ satisfying

$$
\|S(t) f\|_{C_{b}^{l}\left(\mathbb{R}^{N}\right)} \leq C_{k, l} \frac{e^{\omega t}}{t^{(l-k) / 2}}\|f\|_{C_{b}^{k}\left(\mathbb{R}^{N}\right)}, \quad \forall t>0 .
$$

Since the coefficients $q_{i j}$ and $b_{j}(i, j=1, \ldots, N)$ are locally lipschitz continuous, $q_{i j}^{\varepsilon_{n}}$ and $b_{j}^{\varepsilon_{n}}$ converge locally uniformly in $\mathbb{R}^{N}$ as $n$ tends to $+\infty$, respectively to $q_{i j}$ and $b_{j}$, so that $S(t) f$ satisfies the differential equation in (1.1). Moreover, for any $f \in C_{b}^{2}\left(\mathbb{R}^{N}\right), S(t) f$ converges to $f$ as $t$ tends to 0 locally uniformly in $x$. This can be seen by a localization argument. For this purpose, we fix $k \in \mathbb{N}$ and let $\eta=\eta_{k}$ be as in the proof of Theorem 3.3. Moreover, for any $n \in \mathbb{N}$ we 
set $v_{n}(t, x)=\eta(x)\left(T^{\varepsilon_{n}}(t) f\right)(x)$ and observe that $v_{n}$ is a solution to the Cauchy problem

$$
\begin{cases}D_{t} v_{n}(t, x)=\mathcal{A}^{\varepsilon_{n}} v_{n}(t, x)+\psi_{n}(t, x), & (t, x) \in[0, T] \times B(k), \\ v_{n}(t, x)=0, & x \in \partial B(k), \\ v_{n}(0, x)=\eta(x) f(x), & x \in \bar{B}(k),\end{cases}
$$

where

$$
\psi_{n}(t, \cdot)=-2 T^{\varepsilon_{n}}(t) f \cdot \mathcal{A}^{\varepsilon_{n}}(\eta)-2 \sum_{i, j=1}^{N} q_{i j}^{\varepsilon_{n}} D_{i} T^{\varepsilon_{n}}(t) f \cdot D_{j} \eta .
$$

Since the coefficients $q_{i j}^{\varepsilon}$ and $b_{j}^{\varepsilon}(i, j=1, \ldots, N)$ converge locally uniformly in $\mathbb{R}^{N}$, they are equibounded (with respect to $n \in \mathbb{N}$ ) in $B(k)$ and there exists a positive constant $\widetilde{C}$ such that $\left\|\mathcal{A}^{\varepsilon_{n}} g\right\|_{C(\bar{B}(k))} \leq \widetilde{C}\|g\|_{C^{2}(\bar{B}(k))}$ for any $g \in C^{2}(\bar{B}(k))$. Therefore, from (3.30) we deduce that there exists a constant $\bar{C}$, independent of $n$, such that

$$
\left|\psi_{n}(t, x)\right| \leq \frac{\bar{C}}{t^{1 / 2}}\|f\|_{\infty}, \quad \forall(t, x) \in(0, T] \times B(k), n \in \mathbb{N} .
$$

Estimate (3.32) implies that $v_{n}$ can be written by the usual variation-of-constants formula as

$$
v_{n}(t, \cdot)=T_{k, n}(t)(\eta f)+\int_{0}^{t} T_{k, n}(t-s) \psi_{n}(s, \cdot) d s, \quad \forall t \in(0, T],
$$

where $T_{k, n}(t)$ is the semigroup associated with the realization $A_{k, n}$ of $\mathcal{A}^{\varepsilon_{n}}$ in $C(\bar{B}(k))$ with Dirichlet boundary conditions (see Section 2).

Since $\eta f \in D\left(A_{k, n}\right)$ (see (2.6) $)$ and $T_{k, n}$ is a semigroup of contractions in $C(\bar{B}(k)$ ) for any $n \in \mathbb{N}$, then

$$
\begin{aligned}
\left\|T_{k, n}(t)(\eta f)-\eta f\right\|_{C(B(k))} & =\left\|\int_{0}^{t} T_{k, n}(s) \mathcal{A}^{\varepsilon_{n}}(\eta f) d s\right\|_{C(\bar{B}(k))} \\
& \leq t\left\|\mathcal{A}^{\varepsilon_{n}}(\eta f)\right\|_{C(\bar{B}(k))} \leq \bar{C} t\|\eta f\|_{C^{2}(\bar{B}(k))}, \quad \forall t \in(0, T),
\end{aligned}
$$

which readily yields

$$
\lim _{t \rightarrow 0^{+}} \sup _{n \in \mathbb{N}}\left\|T_{k, n}(t)(\eta f)-\eta f\right\|_{C(\bar{B}(k))}=0 .
$$

From (3.32) and 3.33 we can now easily show that

$$
\lim _{t \rightarrow 0^{+}} \sup _{n \in \mathbb{N}}\left\|v_{n}(t, \cdot)-\eta f\right\|_{C(\bar{B}(k))}=0 .
$$

Recalling that $\eta \equiv 1$ in $B(k / 2)$, we deduce that

$$
\begin{aligned}
|(S(t) f)(x)-f(x)| \leq & \sup _{n \in \mathbb{N}}\left|\left(T^{\varepsilon_{n}}(t) f\right)(x)-\eta(x) f(x)\right| \\
& +\limsup _{n \rightarrow+\infty}\left|\left(T^{\varepsilon_{n}}(t) f\right)(x)-(S(t) f)(x)\right| \\
\leq & \sup _{n \in \mathbb{N}}\left\|v_{k}(t, \cdot)-\eta f\right\|_{C(\bar{B}(k))},
\end{aligned}
$$

for any $(t, x) \in(0, T) \times B(k / 2)$. Taking the limit as $t$ tends to 0 in both the first and the last side of (3.34) gives

$$
\lim _{t \rightarrow 0^{+}} \sup _{x \in B(k / 2)}|u(t, x)-f(x)|=0 .
$$


From the arbitrariness of $k \in \mathbb{N}$, we deduce that $S(t) f$ converges locally uniformly (with respect to $x$ ) to $f$ as $t$ tends to 0 . Hence $S(t) f$ is a classical solution to problem (1.1) and the maximum principle in Lemma 2.4 implies that $T(t) f=S(t) f$. Since $C_{b}^{2}\left(\mathbb{R}^{N}\right)$ is dense in $C_{b}^{1}\left(\mathbb{R}^{N}\right)$ (endowed with the sup-norm) we can extend the previous equality to all the $f \in C_{b}^{1}\left(\mathbb{R}^{N}\right)$, obtaining (3.1) in the case when $k \geq 1$.

Finally, with any $f \in C_{b}\left(\mathbb{R}^{N}\right)$ we write $T(t)=T(t / 2) T(t / 2) f$ and observe that since $T(t / 2) f \in C_{b}^{1}\left(\mathbb{R}^{N}\right)$, then $T(t) f=S(t / 2) T(t / 2) f$. Applying (3.31) with $(t, f)$ being replaced by $(t / 2, T(t / 2) f)$, we easily get (3.1) also in the case when $k=0$.

Estimates (3.1) and (3.2) can be used to prove optimal Schauder estimates for the elliptic equation $\lambda u-\mathcal{A} u=f$.

Theorem 3.6. Let $l \in\{1,2\}$ and let assumptions H1-H3, H4-l be satisfied. Then, the maximal domain of the operator $\mathcal{A}$ in $C_{b}\left(\mathbb{R}^{N}\right)$, i.e. the set

$$
D_{\max }(\mathcal{A})=\left\{u \in C_{b}\left(\mathbb{R}^{N}\right) \cap \bigcap_{1<p<+\infty} W_{l o c}^{2, p}\left(\mathbb{R}^{N}\right): \mathcal{A} u \in C_{b}\left(\mathbb{R}^{N}\right)\right\},
$$

is contained in $C_{b}^{\theta}\left(\mathbb{R}^{N}\right)$ for any $\theta \in(0,1]$, if $l=1$, and for any $\theta \in(0,2)$, if $l=2$. Moreover, for any $\omega>0$ and any $\theta$ as above, there exists a positive constant $C=C(\theta, \omega)$ such that

$$
\|u\|_{C_{b}^{\theta}\left(\mathbb{R}^{N}\right)} \leq C\|u\|_{\infty}^{1-\frac{\theta}{2}}\|(\omega-\mathcal{A}) u\|_{\infty}^{\frac{\theta}{2}}, \quad \forall u \in D_{\max }(\mathcal{A}) .
$$

Finally, if assumption H4-3 is also satisfied, then for any $f \in C_{b}^{\alpha}\left(\mathbb{R}^{N}\right)(\alpha \in(0,1))$ and any $\lambda>0$, there exist a unique solution $u \in C_{b}^{2+\alpha}\left(\mathbb{R}^{N}\right)$ of the elliptic equation $\lambda u-\mathcal{A} u=f$ and a positive constant $C=C(\alpha, \lambda)$ such that

$$
\|u\|_{C_{b}^{2+\alpha}\left(\mathbb{R}^{N}\right)} \leq C\|f\|_{C_{b}^{\alpha}\left(\mathbb{R}^{N}\right)} .
$$

Proof. The proof of (3.36) can be obtained from (3.1) arguing as in [12] Theorems 1 and 4.1].

Taking advantage of the quoted estimates and Theorem 3.6, one can now prove sharp estimates for the solution to the Cauchy problem

$$
\left\{\begin{array}{lr}
D_{t} u(t, x)=\mathcal{A} u(t, x)+g(t, x), \quad t>0, & x \in \mathbb{R}^{N}, \\
u(0, x)=u_{0}(x), & x \in \mathbb{R}^{N},
\end{array}\right.
$$

whose "mild" solution is given by the usual variation-of-constants formula

$$
u(t, x)=\left(T(t) u_{0}\right)(x)+\int_{0}^{t}(T(t-s) g(s, \cdot))(x) d s, \quad t>0, x \in \mathbb{R}^{N}
$$

(see e.g. [17] Theorem 3.5]). Arguing as in [12, Theorem 2], one can prove the following theorem.

Theorem 3.7. Suppose that assumptions H1-H3, H4-3 are satisfied. Let T $>0$, $\theta \in(0,1)$ and let $g:[0, T] \times \mathbb{R}^{N} \rightarrow \mathbb{R}$ be a bounded and continuous function such that $g(t, \cdot) \in C_{b}^{\theta}\left(\mathbb{R}^{N}\right)$ for every $t$ and $\sup _{t \in[0, T]}\|g(t, \cdot)\|_{C_{b}^{\theta}\left(\mathbb{R}^{N}\right)}<+\infty$. Moreover, let $u_{0} \in C_{b}^{2+\theta}\left(\mathbb{R}^{N}\right)$. Then, the function $u$ in (3.38) is the unique strict solution to problem (3.37) (i.e. $D_{t} u, D_{x} u, D_{x x} u \in C_{b}\left([0, T] \times \mathbb{R}^{N}\right)$ and $u$ solves (3.37)) and there exists a positive constant $C=C_{T}$ such that

$$
\sup _{t \in[0, T]}\|u(t, \cdot)\|_{C_{b}^{2+\theta}\left(\mathbb{R}^{N}\right)} \leq C\left(\left\|u_{0}\right\|_{C_{b}^{2+\theta}\left(\mathbb{R}^{N}\right)}+\sup _{t \in[0, T]}\|g(t, \cdot)\|_{C_{b}^{\theta}\left(\mathbb{R}^{N}\right)}\right) .
$$




\section{Pointwise estimates}

This section is devoted to prove pointwise estimates for the first-, second- and third-order spatial derivatives of $T(t) f$. Throughout this section we assume that conditions $\mathrm{H} 1-\mathrm{H}_{3}$ of Section 3 hold true and we replace assumptions $\mathrm{H}_{4}$ with the following ones:

H4-1- $\gamma): q_{i j}, b_{j} \in C_{l o c}^{1+\delta}\left(\mathbb{R}^{N}\right)$ for some $\delta \in(0,1)$ and there exist $\gamma \in(0,1)$, a constant $C>0$ and a function $d: \mathbb{R}^{N} \rightarrow \mathbb{R}$ with $\sup _{x \in \mathbb{R}^{N}}\left\{d(x) / \nu(x)^{\gamma}\right\}<$ $+\infty$, such that $\left|D_{k} q_{i j}(x)\right| \leq C \nu(x)^{\gamma}$ for any $i, j, k=1, \ldots, N$ and

$$
\sum_{i, j=1}^{N} D_{i} b_{j}(x) \xi_{i} \xi_{j} \leq d(x)|\xi|^{2}, \quad \forall x, \xi \in \mathbb{R}^{N}
$$

H4-2- $\gamma): q_{i j}, b_{j} \in C_{l o c}^{2+\delta}\left(\mathbb{R}^{N}\right)$ for some $\delta \in(0,1)$, hypothesis $H_{4}-1-\gamma$ holds true and there exist $\gamma \in(0,1)$, a positive function $r: \mathbb{R}^{N} \rightarrow \mathbb{R}$ with

$$
\sup _{x \in \mathbb{R}^{N}}\left\{\left(d(x)+L_{2} r(x)\right) / \nu(x)^{\gamma}\right\}<+\infty,
$$

where $L_{2}:=N^{3 / 2} / \sqrt{8}$, and two constants $C>0, K_{1} \in \mathbb{R}$ such that

$$
\left|D^{\beta} b_{j}(x)\right| \leq r(x), \quad j=1, \ldots, N, \quad|\beta|=2, \quad x \in \mathbb{R}^{N}
$$

and

$$
\sum_{i, j, h, k=1}^{N} D_{h k} q_{i j}(x) m_{i j} m_{h k} \leq K_{1} \nu(x)^{\gamma} \sum_{h, k=1}^{N} m_{h k}^{2}
$$

for any symmetric matrix $M=\left(m_{h k}\right)_{h, k=1}^{N}$ and any $x \in \mathbb{R}^{N}$;

H4-3- $\gamma): q_{i j}, b_{j} \in C_{l o c}^{3+\delta}\left(\mathbb{R}^{N}\right)$ for some $\delta \in(0,1)$, hypothesis $H_{4}-2-\gamma$ holds true with $L_{2}$ being replaced by $L_{3}:=2 / \sqrt{5}$ if $N=1$ and $L_{3}:=\sqrt{N^{3}(N+1) / 3}$ otherwise. Moreover, there exist $\gamma \in(0,1)$ and a constant $C>0$ such that $\left|D^{\beta} b_{j}(x)\right| \leq r(x)$ and $\left|D^{\beta} q_{i j}(x)\right| \leq C \nu(x)^{\gamma}$ for any $i, j=1, \ldots, N$, any $|\beta|=3$ and any $x \in \mathbb{R}^{N}$.

Remark 4.1. Assumptions $H_{4}-l-\gamma(l=1,2,3)$ allow us to consider the case when the coefficients are of polynomial type.

In what follows we denote indifferently by $u$ and $T(t) f$ the solution to problem (2.1) corresponding to the initial datum $f$.

We begin by proving the following lemma which will be essential to prove the first type of pointwise estimates.

Lemma 4.2. Let $k \in\{1,2,3\}$ and let assumptions $H 1-H_{3}$ and $H_{4}$ - $k$ be satisfied. Then, if $f \in C_{b}^{k}\left(\mathbb{R}^{N}\right)$ the function $(t, x) \mapsto\left(D^{k} T(t) f\right)(x)$ is continuous in $[0,+\infty) \times$ $\mathbb{R}^{N}$.

Proof. Let $f \in C_{b}^{k}\left(\mathbb{R}^{N}\right)$ and $u(t, x)=(T(t) f)(x)$. The regularity of $u$ for $t>0$ is a classical result, recalled in Theorem 2.2. Thus we have only to prove the regularity at $t=0$. The proof is based on a localization argument.

Fix $x_{0} \in \mathbb{R}^{N}$, let $\Omega$ be a smooth bounded neighborhood of $x_{0}$ and let $\vartheta \in C_{0}^{\infty}(\Omega)$ be such that $\vartheta \equiv 1$ in a smaller neighborhood $\Omega_{0} \subset \Omega$ of $x_{0}$. Set $v(t, x)=\vartheta(x) u(t, x)$ for $t>0$ and $x \in \Omega$; then the function $v$ satisfies the equation

$$
v_{t}(t, x)-\mathcal{A} v(t, x)=\psi(t, x)=-u(t, x) \mathcal{A} \vartheta(x)-2 \sum_{i, j=1}^{N} q_{i j}(x) D_{i} u(t, x) D_{j} \vartheta(x),
$$


for all $t>0$ and $x \in \Omega$, and the boundary condition $v(t, x)=0$ for all $t>0$ and $x \in \partial \Omega$. Moreover, it is readily seen that there exists a constant $C>0$ such that

$$
\|\psi(t, \cdot)\|_{\infty} \leq C\|u(t, \cdot)\|_{C^{1}(\bar{\Omega})} \leq C \frac{C_{0,1} e^{\omega T}}{\sqrt{t}}\|f\|_{\infty}, \quad 0<t \leq T .
$$

Here, we have used the estimate (3.1) with $(k, l)=(0,1)$. In particular, the function $t \mapsto\|\psi(t, \cdot)\|_{\infty}$ belongs to $L^{1}(0, T)$ for all $T>0$, and therefore we can write

$$
v(t, x)=\left(T_{\Omega}(t)(\vartheta f)\right)(x)=\int_{0}^{t}\left(T_{\Omega}(t-s) \psi(s, \cdot)\right)(x) d s, \quad \forall t>0, x \in \Omega,
$$

where $T_{\Omega}(t)$ is the semigroup associated with the Cauchy problem with Dirichlet boundary condition in $\Omega$. Now let $k=1,2,3$ and $f \in C_{b}^{k}\left(\mathbb{R}^{N}\right)$; using the classical gradient estimates for $T_{\Omega}(t)$ and the estimate (3.1), we get

$$
\begin{aligned}
\left\|D^{k} T_{\Omega}(t-s) \psi(s, \cdot)\right\|_{\infty} & \leq \frac{C_{T}}{\sqrt{t-s}}\|\psi(s, \cdot)\|_{C^{k-1}(\bar{\Omega})} \\
& \leq C \frac{C_{T}}{\sqrt{t-s}}\|u(s, \cdot)\|_{C^{k}(\bar{\Omega})} \\
& \leq C_{k, k} C \frac{C_{T}}{\sqrt{t-s}}\|f\|_{C^{k}\left(\mathbb{R}^{N}\right)},
\end{aligned}
$$

for any $0<s<t \leq T$, where $C, C_{T}>0$ are constants. This means that the function $t \mapsto\left\|D^{k} T_{\Omega}(t-s) \psi(s, \cdot)\right\|_{\infty}$ belongs to $L^{1}(0, T)$ for all $T>0$, and therefore we can write

$$
D^{k} v(t, x)-\left(D^{k} T_{\Omega}(t)(\vartheta f)\right)(x)=\int_{0}^{t}\left(D^{k} T_{\Omega}(t-s) \psi(s, \cdot)\right)(x) d s, \quad t>0, x \in \Omega,
$$

and

$$
\left|D^{k} v(t, x)-\left(D^{k} T_{\Omega}(t)(\vartheta f)\right)(x)\right| \leq \int_{0}^{t} C \frac{C_{T, k}}{\sqrt{t-s}}\|f\|_{C^{k}(\bar{\Omega})} d s, \quad 0<t \leq T, x \in \Omega .
$$

This implies that the function $(t, x) \mapsto D^{k} v(t, x)$ is continuous in $[0, T] \times \Omega_{0}$ since, by virtue of Theorem $2.3\left(D^{k} T_{\Omega}(t)(\vartheta f)\right)(x)$ tends to $D^{k}(\vartheta f)(x)$ uniformly in $\Omega_{\delta}$, for any $\delta>0$, as $t$ tends to 0 . Since $v \equiv u$ in a neighborhood of $x_{0}$, it follows that $D^{k} u(t, x)$ is continuous at $\left(0, x_{0}\right)$.

We now define the following quantities which will be widely used in what follows. We set

$$
M_{1}=\sup _{x \in \mathbb{R}^{N}}\left\{d(x) / \nu(x)^{\gamma}\right\}, \quad M_{k}=\sup _{x \in \mathbb{R}^{N}}\left\{\left(d(x)+L_{k} r(x)\right) / \nu(x)^{\gamma}\right\} \quad(k=2,3),
$$

where $L_{k}(k=2,3)$ is defined in assumption $H_{4}-k-\gamma$.

We are now able to prove the following theorem in which we assume that $M_{k} \geq 0$.

Theorem 4.3. Let $l \in\{1,2,3\}$ and let assumptions H1-H3, H4-l- $\gamma$ be satisfied. Further assume that $M_{l} \geq 0$. Then, for any $f \in C_{b}^{l}\left(\mathbb{R}^{N}\right)$ and any $p \in(1,+\infty)$

$$
\left|\left(D^{l} T(t) f\right)(x)\right|^{p} \leq e^{\sigma_{l, p} t}\left(T(t)\left[\left(\sum_{j=0}^{l}\left|D^{j} f\right|^{2}\right)^{\frac{p}{2}}\right]\right)(x), \quad \forall t>0, x \in \mathbb{R}^{N},
$$


where, if $p \in(1,2]$,

$$
\sigma_{l, p}=p \sup _{x \in \mathbb{R}^{N}}\left\{(1-p) \nu(x)+\frac{C_{l}(p)}{p-1}(\nu(x))^{\gamma}\right\} \vee 0
$$

$C_{l}(p)$ being a suitable positive constant (that can be explicitly determined) such that $\lim _{p \rightarrow 1} C_{l}(p) \in \mathbb{R}$, while, if $p>2, \sigma_{l, p}=\frac{p}{2} \sigma_{l, 2}(l=1,2,3)$.

Proof. We begin with the case when $l=3$ and $p \in(1,2]$. For any $\delta>0$ we introduce the function $w_{\delta}: \overline{\mathbb{R}}_{+} \times \mathbb{R}^{N} \rightarrow \mathbb{R}$ defined by

$$
w_{\delta}(t, x)=\left(|u(t, x)|^{2}+|D u(t, x)|^{2}+\left|D^{2} u(t, x)\right|^{2}+\left|D^{3} u(t, x)\right|^{2}+\delta\right)^{\frac{p}{2}},
$$

for any $t>0$ and any $x \in \mathbb{R}^{N}$. By Lemma 4.2, $w_{\delta} \in C_{b}\left([0,+\infty) \times \mathbb{R}^{N}\right) \cap$ $C_{l o c}^{1,2}\left((0,+\infty) \times \mathbb{R}^{N}\right)$ and a straightforward computation shows that it solves the Cauchy problem

$$
\left\{\begin{array}{lr}
D_{t} w_{\delta}(t, x)=\mathcal{A} w_{\delta}(t, x)+g_{\delta}(t, x), & t>0, \\
w_{\delta}(0, x)=\left(|f(x)|^{2}+|D f(x)|^{2}+\left|D^{2} f(x)\right|^{2}+\left|D^{3} f(x)\right|^{2}+\delta\right)^{\frac{p}{2}}, & x \in \mathbb{R}^{N}
\end{array}\right.
$$

where

$$
\begin{aligned}
& g_{\delta}=p\left(|u|^{2}+|D u|^{2}+\left|D^{2} u\right|^{2}+\left|D^{3} u\right|^{2}+\delta\right)^{\frac{p}{2}-1} \\
& \times\left(-\sum_{i, j=1}^{N} q_{i j} D_{i} u D_{j} u-\sum_{i, j, h=1}^{N} q_{i j} D_{i h} u D_{j h} u-\sum_{i, j, h, k=1}^{N} q_{i j} D_{i h k} u D_{j h k} u\right. \\
& \quad-\sum_{i, j, h, k, l=1}^{N} q_{i j} D_{i h k l} u D_{j h k l} u+\sum_{i, j, h=1}^{N} D_{h} q_{i j} D_{h} u D_{i j} u \\
& \quad+2 \sum_{i, j, h, k=1}^{N} D_{h} q_{i j} D_{h k} u D_{i j k} u+3 \sum_{i, j, h, k, l=1}^{N} D_{h} q_{i j} D_{h k l} u D_{i j k l} u \\
& \quad+\sum_{j, h=1}^{N} D_{h} b_{j} D_{j} u D_{h} u+2 \sum_{j, h, k=1}^{N} D_{h} b_{j} D_{j k} u D_{h k} u \\
& \quad+3 \sum_{j, h, k, l=1}^{N} D_{h} b_{j} D_{j k l} u D_{h k l} u+\sum_{i, j, h, k=1}^{N} D_{h k} q_{i j} D_{i j} u D_{h k} u \\
&+3 \sum_{i, j, h, k, l=1}^{N} D_{h k} q_{i j} D_{i j l} u D_{h k l} u+\sum_{j, h, k=1}^{N} D_{h k} b_{j} D_{j} u D_{h k} u \\
&+3 \sum_{j, h, k, l=1}^{N} D_{h k} b_{j} D_{j l} u D_{h k l} u+\sum_{i, j, h, k, l=1}^{N} D_{h k l} q_{i j} D_{i j} u D_{h k l} u \\
&\left.+\sum_{j, h, k, l=1}^{N} D_{h k l} b_{j} D_{j} u D_{h k l} u\right)
\end{aligned}
$$




$$
\begin{gathered}
+p(2-p)\left(|u|^{2}+|D u|^{2}+\left|D^{2} u\right|^{2}+\left|D^{3} u\right|^{2}+\delta\right)^{\frac{p}{2}-2} \\
\times \sum_{i, j=1}^{N} q_{i j}\left(u D_{i} u+\sum_{h=1}^{N} D_{h} u D_{i h} u+\sum_{h, k=1}^{N} D_{h k} u D_{i h k} u\right. \\
\left.+\sum_{h, k, l=1}^{N} D_{h k l} u D_{i h k l} u\right) \\
\times\left(u D_{j} u+\sum_{h=1}^{N} D_{h} u D_{j h} u+\sum_{h, k=1}^{N} D_{h k} u D_{j h k} u\right. \\
\left.+\sum_{h, k, l=1}^{N} D_{h k l} u D_{j h k l} u\right) .
\end{gathered}
$$

Now, let $h, k \in\{0,1,2,3\}$ be fixed. Applying the Cauchy-Schwarz inequality twice (first to the inner product $(\xi, \eta) \mapsto\langle Q(x) \xi, \eta\rangle$ and then to the euclidean one) we deduce that

$$
\begin{aligned}
& \sum_{i, j=1}^{N} q_{i j} \sum_{|\alpha|=h} D^{\alpha} u D_{i} D^{\alpha} u \sum_{|\beta|=k} D^{\beta} u D_{j} D^{\beta} u \\
= & \sum_{|\alpha|=h|\beta|=k} \sum_{h} D^{\alpha} u D^{\beta} u \sum_{i, j=1}^{N} q_{i j} D_{i} D^{\alpha} u D_{j} D^{\beta} u \\
\leq & \sum_{|\alpha|=h}\left|D^{\alpha} u\right|\left(\sum_{i, j=1}^{N} q_{i j} D_{i} D^{\alpha} u D_{j} D^{\alpha} u\right)^{\frac{1}{2}} \sum_{|\beta|=k}\left|D^{\beta} u\right|\left(\sum_{i, j=1}^{N} q_{i j} D_{i} D^{\beta} u D_{j} D^{\beta} u\right)^{\frac{1}{2}} \\
\leq & \left|D^{h} u\right|\left|D^{k} u\right|\left(\sum_{|\alpha|=h} \sum_{i, j=1}^{N} q_{i j} D_{i} D^{\alpha} u D_{j} D^{\alpha} u\right)^{\frac{1}{2}}\left(\sum_{|\beta|=k} \sum_{i, j=1}^{N} q_{i j} D_{i} D^{\beta} u D_{j} D^{\beta} u\right)^{\frac{1}{2}} .
\end{aligned}
$$

This estimate can be used in order to get

$$
\begin{aligned}
& \sum_{i, j=1}^{N} q_{i j}\left(u D_{i} u+\sum_{h=1}^{N} D_{h} u D_{i h} u+\sum_{h, k=1}^{N} D_{h k} u D_{i h k} u+\sum_{h, k, l=1}^{N} D_{h k l} u D_{i h k l} u\right) \\
& \quad \times\left(u D_{j} u+\sum_{h=1}^{N} D_{h} u D_{j h} u+\sum_{h, k=1}^{N} D_{h k} u D_{j h k} u+\sum_{h, k, l=1}^{N} D_{h k l} u D_{j h k l} u\right) \\
& \leq\left[|u|\left(\sum_{i, j=1}^{N} q_{i j} D_{i} u D_{j} u\right)^{\frac{1}{2}}+|D u|\left(\sum_{i, j, h=1}^{N} q_{i j} D_{i h} u D_{j h} u\right)^{\frac{1}{2}}\right. \\
& \quad+\left|D^{2} u\right|\left(\sum_{i, j, h, k=1}^{N} q_{i j} D_{i h k} u D_{j h k} u\right)^{\frac{1}{2}}
\end{aligned}
$$




$$
\begin{gathered}
\left.+\left|D^{3} u\right|^{2}\left(\sum_{i, j, h, k, l=1}^{N} q_{i j} D_{i h k l} u D_{j h k l} u\right)^{\frac{1}{2}}\right]^{2} \\
\leq\left(|u|^{2}+|D u|^{2}+\left|D^{2} u\right|^{2}+\left|D^{3} u\right|^{2}\right) \\
\times\left(\sum_{i, j=1}^{N} q_{i j} D_{i} u D_{j} u+\sum_{i, j, h=1}^{N} q_{i j} D_{i h} u D_{j h} u\right. \\
\left.\quad+\sum_{i, j, h, k=1}^{N} q_{i j} D_{i h k} u D_{j h k} u+\sum_{i, j, h, k, l=1}^{N} q_{i j} D_{i h k l} u D_{j h k l} u\right) .
\end{gathered}
$$

Taking (4.5) into account, it is immediate to check that

$$
\begin{aligned}
g_{\delta} \leq p\{( & -p)\left[\sum_{i, j=1}^{N} q_{i j} D_{i} u D_{j} u+\sum_{i, j, h=1}^{N} q_{i j} D_{i h} u D_{j h} u\right. \\
& \left.+\sum_{i, j, h, k=1}^{N} q_{i j} D_{i h k} u D_{j h k} u+\sum_{i, j, h, k, l=1}^{N} q_{i j} D_{i h k l} u D_{j h k l} u\right] \\
& +\sum_{i, j, h=1}^{N} D_{h} q_{i j} D_{h} u D_{i j} u+2 \sum_{i, j, h, k=1}^{N} D_{h} q_{i j} D_{h k} u D_{i j k} u \\
& +3 \sum_{i, j, h, k, l=1}^{N} D_{h} q_{i j} D_{h k l} u D_{i j k l} u+\sum_{j, h=1}^{N} D_{h} b_{j} D_{j} u D_{h} u \\
& +2 \sum_{j, h, k=1}^{N} D_{h} b_{j} D_{j k} u D_{h k} u+3 \sum_{j, h, k, l=1}^{N} D_{h} b_{j} D_{j k l} u D_{h k l} u \\
& +\sum_{i, j, h, k=1}^{N} D_{h k} q_{i j} D_{i j} u D_{h k} u+3 \sum_{i, j, h, k, l=1}^{N} D_{h k} q_{i j} D_{i j l} u D_{h k l} u \\
& +\sum_{j, h, k=1}^{N} D_{h k} b_{j} D_{j} u D_{h k} u+3 \sum_{j, h, k, l=1}^{N} D_{h k} b_{j} D_{j l} u D_{h k l} u \\
& \left.+\sum_{i, j, h, k, l=1}^{N} D_{h k l} q_{i j} D_{i j} u D_{h k l} u+\sum_{j, h, k, l=1}^{N} D_{h k l} b_{j} D_{j} u D_{h k l} u\right\} \\
\times & \left(|u|^{2}+|D u|^{2}+\left|D^{2} u\right|^{2}+\left|D^{3} u\right|^{2}+\delta\right)^{\frac{p}{2}-1} .
\end{aligned}
$$

Hence, using hypotheses $H 1$ and $H_{4}-3-\gamma$, we get for all $\varepsilon, \varepsilon_{1}>0$

$$
\begin{gathered}
g_{\delta} \leq p\left\{\left[(1-p) \nu+C \frac{N^{2}}{4 \varepsilon_{0}} \nu^{\gamma}+d+r \frac{N^{2}}{4 \varepsilon_{1}}(N+1)\right]|D u|^{2}\right. \\
+\left[(1-p) \nu+\left(C \varepsilon_{0} N+C \frac{N^{2}}{2 \varepsilon}+C \frac{N^{3}}{4 \varepsilon}+K_{1}\right) \nu^{\gamma}\right. \\
\left.+2 d+r N\left(\varepsilon_{1}+\frac{3 N}{4 \varepsilon_{1}}\right)\right]\left|D^{2} u\right|^{2}
\end{gathered}
$$




$$
\begin{aligned}
& +\left[(1-p) \nu+\left(2 C \varepsilon N+C \frac{3 N^{2}}{4 \varepsilon}+\varepsilon C N^{2}+3 K_{1}\right) \nu^{\gamma}\right. \\
& \left.+3 d+4 r \varepsilon_{1} N\right]\left|D^{3} u\right|^{2} \\
& \left.+\left((1-p) \nu^{1-\gamma}+3 C \varepsilon N\right) \nu^{\gamma}\left|D^{4} u\right|^{2}\right\} \\
& \quad \times\left(|u|^{2}+|D u|^{2}+\left|D^{2} u\right|^{2}+\left|D^{3} u\right|^{2}+\delta\right)^{\frac{p}{2}-1} .
\end{aligned}
$$

We now choose $\varepsilon=\varepsilon_{0}=(p-1) \nu_{0}^{1-\gamma} /(3 C N)$ and $\varepsilon_{1}$ to be the minimazing point of the function $g: \mathbb{R}_{+} \rightarrow \mathbb{R}$ defined by

$$
g(x)=N \max \left\{\left(N^{2}+N\right) /(4 x),\left(4 x^{2}+3 N\right) /(8 x), 4 x / 3\right\}, \quad \forall x \in \mathbb{R}^{N} .
$$

We obtain $\varepsilon_{1}=3 \sqrt{5} / 10$ if $N=1$ and $\varepsilon_{1}=\sqrt{3 N(N+1)} / 4$ otherwise. With this choice of $\varepsilon$ and $\varepsilon_{1}$, we get

$$
\begin{gathered}
g_{\delta} \leq p\left[\left((1-p) \nu+\widetilde{C}_{1}(p) \nu^{\gamma}\right)|D u|^{2}+\left((1-p) \nu+\widetilde{C}_{2}(p) \nu^{\gamma}\right)\left|D^{2} u\right|^{2}\right. \\
\left.+\left((1-p) \nu+\widetilde{C}_{3}(p) \nu^{\gamma}\right)\left|D^{3} u\right|^{2}\right] \\
\quad \times\left(|u|^{2}+|D u|^{2}+\left|D^{2} u\right|^{2}+\left|D^{3} u\right|^{2}+\delta\right)^{\frac{p}{2}-1},
\end{gathered}
$$

where

$$
\begin{aligned}
& \widetilde{C}_{1}(p)=\frac{3 C^{2} N^{3}}{4(p-1)} \nu_{0}^{\gamma-1}+M_{3}, \\
& \widetilde{C}_{2}(p)=\frac{1}{3}(p-1) \nu_{0}^{1-\gamma}+\frac{3 C^{2} N^{3}(N+2)}{4(p-1)} \nu_{0}^{\gamma-1}+K_{1}+2 M_{3}, \\
& \widetilde{C}_{3}(p)=\frac{1}{3}(N+2)(p-1) \nu_{0}^{1-\gamma}+\frac{9 C^{2} N^{3}}{4(p-1)} \nu_{0}^{\gamma-1}+3 K_{1}+3 M_{3},
\end{aligned}
$$

with $M_{3}$ being given by (4.1). Now let $\sigma_{3, p}$ be as in the statement of the theorem with $C_{3}(p)=(p-1) \max \left\{\widetilde{C}_{1}(p), \widetilde{C}_{2}(p), \widetilde{C}_{3}(p)\right\}$. Then, we have

$$
\begin{aligned}
g_{\delta}(t, x) & \leq \sigma_{3, p}\left(|D u|^{2}+\left|D^{2} u\right|^{2}+\left|D^{3} u\right|^{2}\right)\left(|u|^{2}+|D u|^{2}+\left|D^{2} u\right|^{2}+\left|D^{3} u\right|^{2}+\delta\right)^{\frac{p}{2}-1} \\
& \leq \sigma_{3, p} w(t, x),
\end{aligned}
$$

for any $t>0$ and any $x \in \mathbb{R}^{N}$. This yields

$$
D_{t} w_{\delta}(t, x) \leq \mathcal{A} w_{\delta}(t, x)+\sigma_{3, p} w_{\delta}(t, x) .
$$

Now set $z_{\delta}(t, x)=e^{-\sigma_{3, p} t} w_{\delta}(t, x)$ and observe that $z_{\delta}$ satisfies

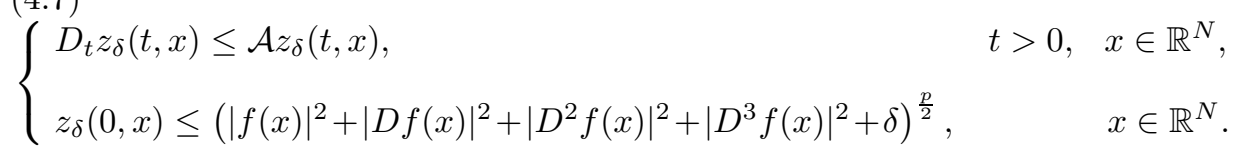


Then, the maximum principle in Lemma 2.4 implies that

$$
z_{\delta}(t, x) \leq\left(T(t)\left(|f|^{2}+|D f|^{2}+\left|D^{2} f\right|^{2}+\left|D^{3} f\right|^{2}\right)^{\frac{p}{2}}\right)(x), \quad t>0, x \in \mathbb{R}^{N},
$$

for any $\delta>0$. Taking the limit as $\delta$ tends to 0 we get

$$
\begin{aligned}
& \left(|u(t, x)|^{2}+|D u(t, x)|^{2}+\left|D^{2} u(t, x)\right|^{2}+\left|D^{3} u(t, x)\right|^{2}\right)^{\frac{p}{2}} \\
\leq & e^{\sigma_{3, p} t}\left(T(t)\left(|f|^{2}+|D f|^{2}+\left|D^{2} f\right|^{2}+\left|D^{3} f\right|^{2}\right)^{\frac{p}{2}}\right)(x),
\end{aligned}
$$

for all $t>0$ and $x \in \mathbb{R}^{N}$. Then, (4.2) follows.

Finally, we consider the case when $p>2$. Since

$$
\begin{aligned}
\left|D^{3} T(t) f(x)\right|^{p} & =\left(\left|\left(D^{3} T(t) f\right)(x)\right|^{2}\right)^{\frac{p}{2}} \\
& \leq\left(e^{\sigma_{3,2} t}\left(T(t)\left(|f|^{2}+|D f|^{2}+\left|D^{2} f\right|^{2}+\left|D^{3} f\right|^{2}\right)\right)(x)\right)^{\frac{p}{2}},
\end{aligned}
$$

we get (4.2) (with $l=3$ ) observing that (2.2) and (2.3) easily imply that

$$
(T(t) \psi)^{p / 2} \leq T(t)\left(\psi^{p / 2}\right),
$$

for any $t>0$ and any nonnegative $\psi \in C_{b}\left(\mathbb{R}^{N}\right)$.

To get (4.2) in the case when $p \in(1,2)$ and $l=1,2$, it suffices to apply the previous arguments to the function

$w_{\delta}(t, x)=\left(|(T(t) f)(x)|^{2}+|(D T(t) f)(x)|^{2}+\delta\right)^{\frac{p}{2}}, \quad t>0, x \in \mathbb{R}^{N}, \delta>0$,

if $l=1$ and to the function

$$
w_{\delta}(t, x)=\left(|(T(t) f)(x)|^{2}+|(D T(t) f)(x)|^{2}+\left.\left(\mid D^{2} T(t) f\right)(x)\right|^{2}+\delta\right)^{\frac{p}{2}}, \delta>0,
$$

if $l=2$. Arguing as above we get

$$
\left(\sum_{j=0}^{l}\left|D^{j} u(t, x)\right|^{2}+\delta\right)^{\frac{p}{2}} \leq e^{\sigma_{l, p} t}\left(T(t)\left(\sum_{j=0}^{l}\left|D^{j} f\right|^{2}+\delta\right)^{\frac{p}{2}}\right)(x)+\delta^{\frac{p}{2}},
$$

for any $l=1,2$, with

$$
\begin{gathered}
\frac{C_{1}(p)}{p-1}=\frac{C^{2} N^{3} \nu_{0}^{\gamma-1}}{4(p-1)}+M_{1} \\
\frac{C_{2}(p)}{p-1}=\max \left\{\frac{C^{2} N^{3} \nu_{0}^{\gamma-1}}{2(p-1)}+M_{2}, \frac{p-1}{2} \nu_{0}^{1-\gamma}+\frac{C^{2} N^{3} \nu_{0}^{\gamma-1}}{p-1}+K_{1}+2 M_{2}\right\},
\end{gathered}
$$

where $M_{1}$ and $M_{2}$ are given by (4.1). Taking the limit as $\delta$ tends to 0 gives (4.2).

In the case when $p>2$, 4.2) (with $l=1,2$ ) follows from the case $p=2$, applying Jensen's inequality. This finishes the proof.

As the following theorem shows, in the case when $M_{k}<0(k=2,3)$ the result in Theorem 4.3 can be improved.

Theorem 4.4. Let $l=1,2,3$ and let assumptions H1-H3, H4-l- $\gamma$ be satisfied. Further, assume that $M_{l}<0($ if $l=2,3)$ or $M_{1} \leq-\frac{C^{2} N^{3}}{4\left(p_{0}-1\right)} \nu_{0}^{\gamma-1}$ for some $p_{0} \in$ $(1,2)$ if $l=1$. Then

$$
\left(\sum_{j=1}^{l}\left|\left(D^{j} T(t) f\right)(x)\right|^{2}\right)^{\frac{p}{2}} \leq e^{\bar{\sigma}_{k, p} t}\left(T(t)\left(\sum_{j=1}^{l}\left|D^{j} f\right|^{2}\right)^{\frac{p}{2}}\right)(x), \quad \forall t>0, x \in \mathbb{R}^{N},
$$


for all $f \in C_{b}^{l}\left(\mathbb{R}^{N}\right)$ and any $p \in(1,+\infty)$ if $l=2,3$ and any $p \in\left[p_{0},+\infty\right)$ if $l=1$, where $\bar{\sigma}_{l, p}(p \in(1,+\infty))$ are suitable constants that can be explicitly determined (see the proof) and they possibly blow up as $p$ tends to 1 .

Proof. The proof is close to that of Theorem 4.3 Hence we just sketch it. To get (4.12), in the case when $p \in(1,2]$ and $l=3$, one has to deal with the function

$$
\bar{w}_{\delta, 3}(t, x)=\left(|D u(t, x)|^{2}+\left|D^{2} u(t, x)\right|^{2}+\left|D^{3} u(t, x)\right|^{2}+\delta\right)^{\frac{p}{2}}, \quad \forall t>0, x \in \mathbb{R}^{N} .
$$

A straightforward computation shows that $\bar{w}_{\delta, 3}$ solves problem (4.3) with $g_{\delta}$ replaced by a function $\bar{g}_{\delta} \in C\left([0,+\infty) \times \mathbb{R}^{N}\right)$ which satisfies estimate (4.6), where we drop out the term $-(1-p) \nu$ in the first round brackets and the term $|u|^{2}$ in the last ones. We now choose $\varepsilon_{0}>-C N^{2} /\left(4 M_{3}\right), \varepsilon$ and $\varepsilon_{1}$ as in the proof of Theorem 4.3, and we set

$$
\begin{aligned}
& \begin{aligned}
C_{1}\left(p, \varepsilon_{0}\right) & =\left(\frac{C N^{2}}{4 \varepsilon_{0}}+M_{3}\right) \nu_{0}^{\gamma}, \\
C_{2}\left(p, \varepsilon_{0}\right) & =\sup _{\mathbb{R}^{N}}\left((1-p) \nu+\left(C \varepsilon_{0} N+\frac{3 C^{2} N^{3}(N+2)}{4(p-1)} \nu_{0}^{\gamma-1}+K_{1}+2 M_{3}\right) \nu^{\gamma}\right), \\
\qquad C_{3}(p) & =\sup _{\mathbb{R}^{N}}\left((1-p) \nu+\widetilde{C}_{3}(p) \nu^{\gamma}\right)
\end{aligned} \\
& \text { and } \bar{\sigma}_{3, p}=p \min _{\varepsilon_{0}>-\frac{C N^{2}}{4 M_{3}}}\left\{\max \left\{C_{1}\left(p, \varepsilon_{0}\right), C_{2}\left(p, \varepsilon_{0}\right), C_{3}(p)\right\}\right\} . \text { We get }
\end{aligned}
$$$$
\begin{aligned}
\bar{g}_{\delta} & \leq \bar{\sigma}_{3, p}\left(|D u|^{2}+\left|D^{2} u\right|^{2}+\left|D^{3} u\right|^{2}\right)\left(|D u|^{2}+\left|D^{2} u\right|^{2}+\left|D^{3} u\right|^{2}+\delta\right)^{\frac{p}{2}-1} \\
& \leq \bar{\sigma}_{3, p} \bar{w}_{\delta, 3}-\bar{\sigma}_{3, p} \delta^{\frac{p}{2}} .
\end{aligned}
$$

If $\bar{\sigma}_{3, p} \geq 0$, the proof follows as in Theorem 4.3. In the case when $\bar{\sigma}_{3, p}<0$, we introduce the function $\bar{z}_{\delta, 3}(t, x)=e^{-\bar{\sigma}_{3, p} t}\left(\bar{w}_{\delta, 3}(t, x)-\delta^{\frac{p}{2}}\right)$ which satisfies (4.7). The maximum principle in Lemma 2.4 implies that

$$
\bar{z}_{\delta, 3}(t, x) \leq\left(T(t)\left(|D f|^{2}+\left|D^{2} f\right|^{2}+\left|D^{3} f\right|^{2}\right)^{\frac{p}{2}}\right)(x),
$$

which gives (4.12) taking the limit as $\delta$ tends to 0 . The case when $p>2$ follows from the case when $p=2$ and from Hölder's inequality, and gives $\bar{\sigma}_{3, p}=\frac{p}{2} \bar{\sigma}_{3,2}$.

Applying the same arguments respectively to the functions

$$
\bar{w}_{\delta, 1}=\left(|D u|^{2}+\delta\right)^{\frac{p}{2}}, \quad \bar{w}_{\delta, 2}=\left(|D u|^{2}+\left|D^{2} u\right|^{2}+\delta\right)^{\frac{p}{2}},
$$

one finally gets (4.12) with $\bar{\sigma}_{1, p}=p\left(p_{0}-p\right) \nu_{0}$ and

$$
\bar{\sigma}_{2, p}=p \min _{\varepsilon_{0}>-\frac{C N^{2}}{4 M_{2}}}\left\{\max \left\{E_{1}\left(\varepsilon_{0}\right), E_{2}\left(\varepsilon_{0}\right)\right\}\right\}
$$

where

$$
E_{1}\left(\varepsilon_{0}\right)=\left(\frac{C N^{2}}{4 \varepsilon_{0}}+M_{2}\right) \nu_{0}^{\gamma}
$$

and

$$
E_{2}\left(\varepsilon_{0}\right)=\sup _{\mathbb{R}^{N}}\left[(1-p) \nu+\left(C \varepsilon_{0} N+\frac{C^{2} N^{3}}{p-1} \nu_{0}^{\gamma-1}+K_{1}+2 M_{2}\right) \nu^{\gamma}\right],
$$

if $p \in(1,2]$, and $\bar{\sigma}_{l, p}=\frac{p}{2} \bar{\sigma}_{l, 2}(l=1,2)$ if $p>2$.

Remark 4.5. In the case when $l=1$, estimate (4.12) has already been proved in [4] under assumption $H_{4}-1-\gamma$ with $\gamma=1 / 2$ for any value of the constant $M_{1}$. 
We now consider the second type of pointwise estimates we want to prove. First we prove the following lemma.

Lemma 4.6. If $g_{R}, g:[0, T] \times \mathbb{R}^{N}, R>0$, are continuous functions such that $\left\|g_{R}\right\|_{\infty} \leq M$ for all $R>0$ and $\lim _{R \rightarrow+\infty} g_{R}=g$, uniformly in $[\varepsilon, T-\varepsilon] \times B(k)$ for every $\varepsilon, k>0$, then

$$
\lim _{R \rightarrow+\infty}\left(T_{R}(t) g_{R}(t)\right)(x)=(T(t) g(t))(x),
$$

uniformly in $[\varepsilon, T-\varepsilon] \times B(k)$ for every $\varepsilon, k>0$.

If $f_{\varepsilon}, f_{0} \in C_{b}\left(\mathbb{R}^{N}\right), \varepsilon>0$, are such that $\left\|f_{\varepsilon}\right\|_{\infty} \leq M$ for all $\varepsilon>0$ and $\lim _{\varepsilon \rightarrow 0^{+}} f_{\varepsilon}=f_{0}$, uniformly in $B(k)$ for all $k>0$, then for all $t>0$ we have $\lim _{\varepsilon \rightarrow 0^{+}} T(t-\varepsilon) f_{\varepsilon}=T(t) f_{0}$, uniformly in $B(k)$ for all $k>0$.

Proof. Let $g_{R}, g$ as above. We have

$$
\begin{aligned}
\left|T_{R}(t) g_{R}(t)-T(t) g(t)\right| & \leq\left|T_{R}(t)\left(g_{R}(t)-g(t)\right)\right|+\left|\left(T(t)-T_{R}(t)\right) g(t)\right| \\
& \leq T_{R}(t)\left(\left|g_{R}(t)-g(t)\right|\right)+\left(T(t)-T_{R}(t)\right)|g(t)| \\
& \leq T(t)\left(\left|g_{R}(t)-g(t)\right|\right)+M\left(T(t)-T_{R}(t)\right) \mathbb{1},
\end{aligned}
$$

for all $R>0$. In deriving (4.13) we took advantage of (2.9).

Let us observe that the second term in the last part of (4.13) converges to zero uniformly in $[\varepsilon, T] \times B(k)$ for every $\varepsilon, k>0$, by (2.7). To estimate the first term, we observe that for any $t \in[\varepsilon, T-\varepsilon]$ and any $x \in \mathbb{R}^{N}$ we have

$$
\left(T(t)\left(\left|g_{R}(t)-g(t)\right|\right)\right)(x) \leq\left(T(t)\left(\sup _{t \in[\varepsilon, T-\varepsilon]}\left|g_{R}(t)-g(t)\right|\right)\right)(x) .
$$

Since $\sup _{t \in[\varepsilon, T-\varepsilon]}\left|g_{R}(t)-g(t)\right|$ is a bounded and continuous function in $\mathbb{R}^{N}$, converging to 0 , locally uniformly in $\mathbb{R}^{N}$, from (2.4) we deduce that the right-hand side of (4.14) converges to 0 , locally uniformly, as $R$ tends to $+\infty$, and the conclusion follows.

We prove the second statement. Let $f_{\varepsilon}, f_{0}$ be as above; we have

$$
\left|T(t-\varepsilon) f_{\varepsilon}-T(t) f_{0}\right| \leq\left|T(t-\varepsilon) f_{\varepsilon}-T(t-\varepsilon) f_{0}\right|+\left|T(t-\varepsilon) f_{0}-T(t) f_{0}\right|
$$

for all $\varepsilon>0$. By (2.4), for any fixed $t>0$, the first term in (4.15) converges to 0 as $\varepsilon$ tends to 0 , locally uniformly in $B(k)$ for any $k>0$. The second term in the right-hand side of (4.15) converges to zero uniformly in $B(k)$ for any $k>0$ as well, since the function $T(t) f_{0}$ is continuous in $[0,+\infty) \times \mathbb{R}^{N}$ and, consequently, uniformly continuous in $[0, T] \times B(k)$ for any $T, k>0$.

We can now prove our estimates. For notation convenience, we set

$\hat{\sigma}_{1, p}=\left\{\begin{array}{ll}\sigma_{1, p}, & \text { if } M_{1} \geq-\frac{C^{2} N^{3}}{4\left(p_{0}-1\right)} \nu_{0}^{\gamma-1} \text { and } p \geq p_{0}, \\ \bar{\sigma}_{1, p}, & \text { otherwise, }\end{array} \quad \hat{\sigma}_{l, p}= \begin{cases}\sigma_{l, p}, & \text { if } M_{l} \geq 0, \\ \bar{\sigma}_{l, p}, & \text { otherwise, }\end{cases}\right.$ for $l=2,3$, where $\sigma_{l, p}$ and $\bar{\sigma}_{l, p}$ are defined, respectively, in Theorems 4.3 and 4.4 .

Theorem 4.7. Let $l \in\{1,2,3\}$ and suppose that assumptions H1-H3, and H4-l- $\gamma$ hold. Then, for any $f \in C_{b}^{l-1}\left(\mathbb{R}^{N}\right)$ we have

$$
\left|\left(D^{l} T(t) f\right)(x)\right|^{p} \leq\left(\frac{\hat{\sigma}_{l, p \wedge 2}}{1-e^{-\hat{\sigma}_{l, p \wedge 2} t}} \phi_{l, p \wedge 2}(t)\right)^{\max \left\{1, \frac{p}{2}\right\}}\left(T(t)\left(\sum_{j=0}^{l-1}\left|D^{j} f\right|^{2}\right)^{\frac{p}{2}}\right)(x),
$$


for any $t>0$ and $x \in \mathbb{R}^{N}$, where $\phi_{1, r}(t)=\left(r(r-1) \nu_{0}\right)^{-\frac{r}{2}} t^{1-\frac{r}{2}}+t$ for any $r \in(1,2]$, and

$$
\phi_{k, r}(t)=a_{r, k}\left(\frac{e^{\hat{\sigma}_{k-1, r} t}-1}{\hat{\sigma}_{k-1, r}}\right)^{1-\frac{r}{2}}+t, \quad r \in(1,2], \quad k=2,3,
$$

$a_{k, r}(r \in(1,2], k=2,3)$ being positive constants that can be explicitly determined from the data.

Proof. We first consider the case when $l=3$ and $p \in(1,2)$. Even if we limit ourselves to the case when $M_{3} \geq 0$, our techniques can also be used, with minor changes, in the case when $M_{3}<0$.

We fix $\delta, t, R>0$ and let $\eta_{R}$ be the cut-off function defined at the beginning of the proof of Theorem 3.3 For any $\alpha, \beta>0$ and any $f \in C_{b}^{2}\left(\mathbb{R}^{N}\right)$ we define the function $g_{\delta}:[0, t] \rightarrow C(B(R))$ by

$$
\begin{aligned}
g_{\delta}(s)=T_{R}(s) & \left(\left(\alpha\left|T_{R}(t-s) f\right|^{2}+\beta \eta_{R}^{2}\left|D T_{R}(t-s) f\right|^{2}\right.\right. \\
& \left.\left.+\eta_{R}^{4}\left|D^{2} T_{R}(t-s) f\right|^{2}+\delta\right)^{\frac{p}{2}}-\delta^{\frac{p}{2}}\right), \quad 0 \leq s \leq t,
\end{aligned}
$$

where $T_{R}(\cdot)$ is the semigroup generated in $C_{b}(\bar{B}(R))$ by the realization $A_{R}$ of the operator $\mathcal{A}$ with Dirichlet boundary conditions (see Section 2 ). To simplify the notation, throughout the remainder of the proof, we set $\phi_{R}^{r}:=T_{R}(t-r) f$ and $\phi^{r}:=T(t-r) f$ for any $r \in[0, t]$.

As is easily seen the function

$$
\left(\alpha\left|\phi_{R}^{r}\right|^{2}+\beta \eta_{R}^{2}\left|D \phi_{R}^{r}\right|^{2}+\eta_{R}^{4}\left|D^{2} \phi_{R}^{r}\right|^{2}+\delta\right)^{p / 2}-\delta^{p / 2}
$$

belongs to $D\left(A_{R}\right)$ for any $r \in[0, t)$. Recalling that $\mathcal{A}\left(\delta^{p / 2}\right)=0$, we get

$$
\begin{aligned}
g_{\delta}^{\prime}(s)=p T_{R}(s)\{ & {\left[\alpha \sum_{i, j=1}^{N} q_{i j} D_{i} \phi_{R}^{s} D_{j} \phi_{R}^{s}+\beta \eta_{R}^{2} \sum_{i, j, h=1}^{N} q_{i j} D_{i h} \phi_{R}^{s} D_{j h} \phi_{R}^{s}\right.} \\
& +\eta_{R}^{4} \sum_{i, j, h, k=1}^{N} q_{i j} D_{i h k} \phi_{R}^{s} D_{j h k} \phi_{R}^{s}+\frac{\beta}{2} \mathcal{A}\left(\eta_{R}^{2}\right)\left|D \phi_{R}^{s}\right|^{2} \\
& +\frac{1}{2} \mathcal{A}\left(\eta_{R}^{4}\right)\left|D^{2} \phi_{R}^{s}\right|^{2}+4 \beta \eta_{R} \sum_{i, j, h=1}^{N} q_{i j} D_{i} \eta_{R} D_{h} \phi_{R}^{s} D_{j h} \phi_{R}^{s} \\
& +8 \eta_{R}^{3} \sum_{i, j, h, k=1}^{N} q_{i j} D_{j} \eta_{R} D_{h k} \phi_{R}^{s} D_{i h k} \phi_{R}^{s} \\
& -\beta \eta_{R}^{2}\left(\sum_{i, j, h=1}^{N} D_{h} q_{i j} D_{h} \phi_{R}^{s} D_{i j} \phi_{R}^{s}+\sum_{j, h=1}^{N} D_{h} b_{j} D_{j} \phi_{R}^{s} D_{h} \phi_{R}^{s}\right)
\end{aligned}
$$




$$
\begin{gathered}
-\eta_{R}^{4}\left(\sum_{i, j, h, k=1}^{N}\left(2 D_{h} q_{i j} D_{i j k} \phi_{R}^{s}+D_{h k} q_{i j} D_{i j} \phi_{R}^{s}\right) D_{h k} \phi_{R}^{s}\right. \\
\left.\left.+\sum_{j, h, k=1}^{N}\left(2 D_{h} b_{j} D_{j k} \phi_{R}^{s}+D_{h k} b_{j} D_{j} \phi_{R}^{s}\right) D_{h k} \phi_{R}^{s}\right)\right] \\
\left.\times\left(\alpha\left|\phi_{R}^{s}\right|^{2}+\beta \eta_{R}^{2}\left|D \phi_{R}^{s}\right|^{2}+\eta_{R}^{4}\left|D^{2} \phi_{R}^{s}\right|^{2}+\delta\right)^{\frac{p}{2}-1}\right\} \\
-\frac{p(2-p)}{4} T_{R}(s)\left[\sum_{i, j=1}^{N} q_{i j} D_{i}\left(\alpha\left|\phi_{R}^{s}\right|^{2}+\beta \eta_{R}^{2}\left|D \phi_{R}^{s}\right|^{2}+\eta_{R}^{4}\left|D^{2} \phi_{R}^{s}\right|^{2}\right)\right. \\
\left.\times\left(\alpha\left|\phi_{R}^{s}\right|^{2}+\beta \eta_{R}^{2}\left|D \phi_{R}^{s}\right|^{2}+\eta_{R}^{4}\left|D^{2} \phi_{R}^{s}\right|^{2}+\delta\right)^{\frac{p}{2}-2}\right] .
\end{gathered}
$$

Arguing as in the proof of (4.4) and recalling the inequality $(a+b)^{2} \leq(1+\varepsilon) a^{2}+$ $\left(1+\varepsilon^{-1}\right) b^{2}$ for any $a, b, \varepsilon>0$, we easily deduce that

$$
\begin{aligned}
& \frac{1}{4} \sum_{i, j=1}^{N} q_{i j} D_{i}\left(\alpha\left(\phi_{R}^{s}\right)^{2}+\beta \eta_{R}^{2}\left|D \phi_{R}^{s}\right|^{2}+\eta_{R}^{4}\left|D^{2} \phi_{R}^{s}\right|^{2}\right) \\
& \times D_{j}\left(\alpha\left(\phi_{R}^{s}\right)^{2}+\beta \eta_{R}^{2}\left|D \phi_{R}^{s}\right|^{2}+\eta_{R}^{4}\left|D^{2} \phi_{R}^{s}\right|^{2}\right) \\
& \leq(1+\varepsilon)\left[\alpha\left|\phi_{R}^{s}\right|\left(\sum_{i, j=1}^{N} q_{i j} D_{i} \phi_{R}^{s} D_{j} \phi_{R}^{s}\right)^{\frac{1}{2}}+\beta \eta_{R}^{2}\left|D \phi_{R}^{s}\right|\left(\sum_{i, j, h=1}^{N} q_{i j} D_{i h} \phi_{R}^{s} D_{j h} \phi_{R}^{s}\right)^{\frac{1}{2}}\right. \\
& \left.+\eta_{R}^{4}\left|D^{2} \phi_{R}^{s}\right|\left(\sum_{i, j, h, k=1}^{N} q_{i j} D_{i h k} \phi_{R}^{s} D_{j h k} \phi_{R}^{s}\right)^{\frac{1}{2}}\right]^{2} \\
& +\left(1+\frac{1}{\varepsilon}\right)\left(\beta \eta_{R}\left|D \phi_{R}^{s}\right|^{2}+2 \eta_{R}^{3}\left|D^{2} \phi_{R}^{s}\right|^{2}\right)^{2} \sum_{i, j=1}^{N} q_{i j} D_{i} \eta_{R} D_{j} \eta_{R} \\
& \leq(1+\varepsilon)\left[\alpha \sum_{i, j=1}^{N} q_{i j} D_{i} \phi_{R}^{s} D_{j} \phi_{R}^{s}+\beta \eta_{R}^{2} \sum_{i, j, h=1}^{N} q_{i j} D_{i h} \phi_{R}^{s} D_{j h} \phi_{R}^{s}\right. \\
& \left.+\eta_{R}^{4} \sum_{i, j, h, k=1}^{N} q_{i j} D_{i h k} \phi_{R}^{s} D_{j h k} \phi_{R}^{s}\right]
\end{aligned}
$$




$$
\begin{aligned}
& \text { EStimates of the DeRivatives For Parabolic operators } \\
& \times\left(\alpha\left|\phi_{R}^{s}\right|^{2}+\beta \eta_{R}^{2}\left|D \phi_{R}^{s}\right|^{2}+\eta_{R}^{4}\left|D^{2} \phi_{R}^{s}\right|^{2}\right) \\
& +\left(1+\frac{1}{\varepsilon}\right)\left(\beta\left|D \phi_{R}^{s}\right|^{2}+4 \eta_{R}^{2}\left|D^{2} \phi_{R}^{s}\right|^{2}\right)\left(\beta \eta_{R}^{2}\left|D \phi_{R}^{s}\right|^{2}+\eta_{R}^{4}\left|D^{2} \phi_{R}^{s}\right|^{2}\right) \\
& \times \sum_{i, j=1}^{N} q_{i j} D_{i} \eta_{R} D_{j} \eta_{R} .
\end{aligned}
$$

Hence,

$$
\begin{aligned}
& g_{\delta}^{\prime}(s) \geq p T_{R}(s)\left\{\left[\alpha C_{\varepsilon, p} \sum_{i, j=1}^{N} q_{i j} D_{i} \phi_{R}^{s} D_{j} \phi_{R}^{s}+\beta C_{\varepsilon, p} \eta_{R}^{2} \sum_{i, j, h=1}^{N} q_{i j} D_{i h} \phi_{R}^{s} D_{j h} \phi_{R}^{s}\right.\right. \\
& +C_{\varepsilon, p} \eta_{R}^{4} \sum_{i, j, h, k=1}^{N} q_{i j} D_{i h k} \phi_{R}^{s} D_{j h k} \phi_{R}^{s}+\frac{\beta}{2} \mathcal{A}\left(\eta_{R}^{2}\right)\left|D \phi_{R}^{s}\right|^{2} \\
& +\frac{1}{2} \mathcal{A}\left(\eta_{R}^{4}\right)\left|D^{2} \phi_{R}^{s}\right|^{2}+4 \beta \eta_{R} \sum_{i, j, h=1}^{N} q_{i j} D_{i} \eta_{R} D_{h} \phi_{R}^{s} D_{j h} \phi_{R}^{s} \\
& +8 \eta_{R}^{3} \sum_{i, j, h, k=1}^{N} q_{i j} D_{j} \eta_{R} D_{h k} \phi_{R}^{s} D_{i h k} \phi_{R}^{s} \\
& -\beta \eta_{R}^{2}\left(\sum_{i, j, h=1}^{N} D_{h} q_{i j} D_{h} \phi_{R}^{s} D_{i j} \phi_{R}^{s}+\sum_{j, h=1}^{N} D_{h} b_{j} D_{j} \phi_{R}^{s} D_{h} \phi_{R}^{s}\right) \\
& -\eta_{R}^{4}\left(\sum_{i, j, h, k=1}^{N}\left(2 D_{h} q_{i j} D_{i j k} \phi_{R}^{s}+D_{h k} q_{i j} D_{i j} \phi_{R}^{s}\right) D_{h k} \phi_{R}^{s}\right. \\
& \left.+\sum_{j, h, k=1}^{N}\left(2 D_{h} b_{j} D_{j k} \phi_{R}^{s}+D_{h k} b_{j} D_{j} \phi_{R}^{s}\right) D_{h k} \phi_{R}^{s}\right) \\
& -(2-p)\left(1+\frac{1}{\varepsilon}\right)\left(\beta\left|D \phi_{R}^{s}\right|^{2}+4 \eta_{R}^{2}\left|D^{2} \phi_{R}^{s}\right|^{2}\right) \\
& \left.\times \sum_{i, j=1}^{N} q_{i j} D_{i} \eta_{R} D_{j} \eta_{R}\right] \\
& \left.\times\left(\alpha\left|\phi_{R}^{s}\right|^{2}+\beta \eta_{R}^{2}\left|D \phi_{R}^{s}\right|^{2}+\eta_{R}^{4}\left|D^{2} \phi_{R}^{s}\right|^{2}+\delta\right)^{\frac{p}{2}-1}\right\},
\end{aligned}
$$


where $C_{\varepsilon, p}=1+(1+\varepsilon)(p-2)$. Taking assumption $H 3$ into account and arguing as in the proof of (3.18), one can easily check that

$$
\sum_{i, j=1}^{N} q_{i j}(x) D_{i} \eta_{R}(x) D_{j} \eta_{R}(x) \leq C^{\prime \prime \prime} \nu(x)
$$

for any $x \in B(R)$ and some positive constant $C^{\prime \prime \prime}$, independent of $R$. Moreover, in view of (3.18)-(3.20) we can write

$$
\mathcal{A}\left(\eta_{R}^{2}\right) \geq-2\left(C^{\prime}+C^{\prime \prime}\right) \nu
$$

$$
\begin{gathered}
\mathcal{A}\left(\eta_{R}^{4}\right)=2 \eta_{R}^{2} \mathcal{A}\left(\eta_{R}^{2}\right)+2 \sum_{i, j=1}^{N} q_{i j} D_{i} \eta_{R}^{2} D_{j} \eta_{R}^{2} \geq 2 \eta_{R}^{2} \mathcal{A}\left(\eta_{R}^{2}\right) \geq-4\left(C^{\prime}+C^{\prime \prime}\right) \eta_{R}^{2} \nu \\
\text {.21) }\left|\eta_{R} \sum_{i, j, h=1}^{N} q_{i j} D_{i} \eta_{R} D_{h} \phi_{R}^{s} D_{j h} \phi_{R}^{s}\right| \leq \frac{C^{\prime \prime} N}{4 \varepsilon} \nu\left|D \phi_{R}^{s}\right|^{2}+\varepsilon C^{\prime \prime} \eta_{R}^{2} \nu\left|D^{2} \phi_{R}^{s}\right|^{2}
\end{gathered}
$$

$$
\left|\eta_{R}^{3} \sum_{i, j, h, k=1}^{N} q_{i j} D_{j} \eta_{R} D_{h k} \phi_{R}^{s} D_{i h k} \phi_{R}^{s}\right| \leq \frac{C^{\prime \prime} N}{4 \varepsilon} \eta_{R}^{2} \nu\left|D^{2} \phi_{R}^{s}\right|^{2}+\varepsilon C^{\prime \prime} \eta_{R}^{4} \nu\left|D^{3} \phi_{R}^{s}\right|^{2},
$$

for any $\varepsilon>0$.

Now, from assumptions $H 1, H_{4}-1-\gamma$ and (4.18), (4.19)-(4.22), we easily deduce that

$$
\begin{aligned}
& g_{\delta}^{\prime}(s) \geq p T_{R}(s)\{[\left(\left(\alpha C_{\varepsilon, p}-\beta\left(C^{\prime}+C^{\prime \prime}\right)-\beta \frac{C^{\prime \prime} N}{\varepsilon}+\beta(p-2) C^{\prime \prime \prime}\left(1+\frac{1}{\varepsilon}\right)\right) \nu\right. \\
&\left.-\beta \frac{C N^{2}}{4 \varepsilon} \nu^{\gamma}-\eta_{R}^{2}\left(\beta d+r \frac{N^{2}}{4 \varepsilon}\right)\right)\left|D \phi_{R}^{s}\right|^{2} \\
&+\left(\left(\beta\left(C_{\varepsilon, p}-4 \varepsilon C^{\prime \prime}\right)-2\left(C^{\prime}+C^{\prime \prime}\right)-\frac{2 C^{\prime \prime} N}{\varepsilon}\right.\right. \\
&+\left.4(p-2) C^{\prime \prime \prime}\left(1+\frac{1}{\varepsilon}\right)\right) \nu \\
&-\left.\left(\beta C \varepsilon N+\frac{C N^{2}}{2 \varepsilon}+K_{1}^{+}\right) \nu^{\gamma}-\eta_{R}^{2}(2 d+r \varepsilon N)\right) \eta_{R}^{2}\left|D^{2} \phi_{R}^{s}\right|^{2} \\
&+\left.\left(\left(C_{\varepsilon, p}-8 \varepsilon C^{\prime \prime}\right) \nu-2 C \varepsilon N \nu^{\gamma}\right) \eta_{R}^{4}\left|D^{3} \phi_{R}^{s}\right|^{2}\right] \\
&\left.\times\left(\alpha\left|\phi_{R}^{s}\right|^{2}+\beta \eta_{R}^{2}\left|D \phi_{R}^{s}\right|^{2}+\eta_{R}^{4}\left|D^{2} \phi_{R}^{s}\right|^{2}+\delta\right)^{\frac{p}{2}-1}\right\} .
\end{aligned}
$$

In view of assumption $\mathrm{H}_{4}-3-\gamma$, we have

$$
\begin{aligned}
& \beta d(x)+r(x) \frac{N^{2}}{4 \varepsilon} \leq\left(\beta-\frac{N^{2}}{4 \varepsilon} L_{3}^{-1}\right) M_{3}^{+}(\nu(x))^{\gamma}+\frac{N^{2}}{4 \varepsilon} L_{3}^{-1} M_{3}^{+}(\nu(x))^{\gamma}, \\
& 2 d(x)+\varepsilon r(x) N \leq 2 M_{3}^{+}(\nu(x))^{\gamma},
\end{aligned}
$$


for any $x \in \mathbb{R}^{N}$, provided $\varepsilon<2 L_{3} N^{-1}$ and $\beta>N^{2} /\left(4 \varepsilon L_{3}\right)$. Here $L_{3}$ is as in assumption $H_{4}-3-\gamma$ and $M_{3}$ is given by (4.1).

We now choose $\varepsilon$ sufficiently small and $\alpha=\alpha_{p}, \beta=\beta_{p}$ sufficiently large such that

$$
\left\{\begin{array}{c}
0<A_{1}:=\alpha(1+(1+\varepsilon)(p-2))-\beta\left(C^{\prime}+C^{\prime \prime}\right) \\
-\beta C^{\prime \prime} N \varepsilon^{-1}+\beta(p-2) C^{\prime \prime \prime}\left(1+\varepsilon^{-1}\right), \\
0<A_{2}:=\beta\left(1+(1+\varepsilon)(p-2)-4 \varepsilon C^{\prime \prime}\right)-2\left(C^{\prime}+C^{\prime \prime}\right) \\
\quad-2 C^{\prime \prime} N \varepsilon^{-1}+4(p-2) C^{\prime \prime \prime}\left(1+\varepsilon^{-1}\right), \\
\left(\beta C N^{2} \varepsilon^{-1}+\left(4 \beta-N^{2} \varepsilon^{-1} L_{3}^{-1}\right) M_{3}^{+}+N^{2} \varepsilon^{-1} L_{3}^{-1} M_{3}^{+}\right) \nu_{0}^{\gamma-1}<4 A_{1}, \\
\left(2 \beta C \varepsilon N+C N^{2} \varepsilon^{-1}+2 K_{1}^{+}+4 M_{3}^{+}\right) \nu_{0}^{\gamma-1}<2 A_{2}, \\
0<A_{3}:=1+(p-2)(1+\varepsilon)-8 \varepsilon C^{\prime \prime}, \quad 2 C \varepsilon N \nu_{0}^{\gamma-1}<A_{3}, \\
\varepsilon<2 L_{3} N^{-1}, \quad 4 \beta>N^{2} L_{3}^{-1} \varepsilon^{-1} .
\end{array}\right.
$$

Consequently, we can find out a positive constant $c_{p}$ such that

$$
\begin{aligned}
g_{\delta}^{\prime}(s) \geq c_{p} T_{R}(s) & \left(\eta_{R}^{4}\left(\left|D \phi_{R}^{s}\right|^{2}+\left|D^{2} \phi_{R}^{s}\right|^{2}+\left|D^{3} \phi_{R}^{s}\right|^{2}\right)\right. \\
& \left.\times\left(\alpha_{p}\left|\phi_{R}^{s}\right|^{2}+\beta_{p} \eta_{R}^{2}\left|D \phi_{R}^{s}\right|^{2}+\eta_{R}^{4}\left|D^{2} \phi_{R}^{s}\right|^{2}+\delta\right)^{\frac{p}{2}-1}\right) .
\end{aligned}
$$

Then, integrating (4.23) in $[\varepsilon, t-\varepsilon](\varepsilon>0)$ we get

$$
\begin{aligned}
& c_{p} \int_{\varepsilon}^{t-\varepsilon}\left(T _ { R } ( s ) \left(\eta_{R}^{4}\left(\left|D \phi_{R}^{s}\right|^{2}+\left|D^{2} \phi_{R}^{s}\right|^{2}+\left|D^{3} \phi_{R}^{s}\right|^{2}\right)\right.\right. \\
& \left.\left.\times\left(\alpha_{p}\left|\phi_{R}^{s}\right|^{2}+\beta_{p} \eta_{R}^{2}\left|D \phi_{R}^{s}\right|^{2}+\eta_{R}^{4}\left|D^{2} \phi_{R}^{s}\right|^{2}+\delta\right)^{\frac{p}{2}-1}\right)\right)(x) d s \\
\leq & \left(T_{R}(t-\varepsilon)\left(\left(\alpha_{p}\left|T_{R}(\varepsilon) f\right|^{2}+\beta_{p} \eta_{R}^{2}\left|D T_{R}(\varepsilon) f\right|^{2}+\eta_{R}^{4}\left|D^{2} T_{R}(\varepsilon) f\right|^{2}+\delta\right)^{\frac{p}{2}}-\delta^{\frac{p}{2}}\right)\right)(x) \\
& -\left(T_{R}(\varepsilon)\left(\left(\alpha_{p}\left|\phi_{R}^{\varepsilon}\right|^{2}+\beta_{p} \eta_{R}^{2}\left|D \phi_{R}^{\varepsilon}\right|^{2}+\eta_{R}^{4}\left|D^{2} \phi_{R}^{\varepsilon}\right|^{2}+\delta\right)^{\frac{p}{2}}-\delta^{\frac{p}{2}}\right)\right)(x) \\
\leq & \left(T_{R}(t-\varepsilon)\left(\left(\alpha_{p}\left|T_{R}(\varepsilon) f\right|^{2}+\beta_{p} \eta_{R}^{2}\left|D T_{R}(\varepsilon) f\right|^{2}+\eta_{R}^{4}\left|D^{2} T_{R}(\varepsilon) f\right|^{2}\right)^{\frac{p}{2}}\right)\right)(x) .
\end{aligned}
$$

Therefore, taking (2.11) into account, from Lemma 4.6 we have

$$
\begin{aligned}
\lim _{R \rightarrow+\infty}\left\{T _ { R } ( s ) \left[\eta_{R}^{4}\left(\left|D \phi_{R}^{s}\right|^{2}+\left|D^{2} \phi_{R}^{s}\right|^{2}+\left|D^{3} \phi_{R}^{s}\right|^{2}\right)\right.\right. \\
\left.\left.\times\left(\alpha_{p}\left|\phi_{R}^{s}\right|^{2}+\beta_{p} \eta_{R}^{2}\left|D \phi_{R}^{s}\right|^{2}+\eta_{R}^{4}\left|D^{2} \phi_{R}^{s}\right|^{2}+\delta\right)^{\frac{p}{2}-1}\right]\right\}(x) \\
=\left\{T ( s ) \left[\left(\left|D \phi^{s}\right|^{2}+\left|D^{2} \phi^{s}\right|^{2}+\left|D^{3} \phi^{s}\right|^{2}\right)\right.\right. \\
\left.\left.\times\left(\alpha_{p}\left|\phi^{s}\right|^{2}+\beta_{p}\left|D \phi^{s}\right|^{2}+\left|D^{2} \phi^{s}\right|^{2}+\delta\right)^{\frac{p}{2}-1}\right]\right\}(x),
\end{aligned}
$$

the convergence being uniform in $[\varepsilon, t-\varepsilon] \times B(k)$ for any $k>0$, and

$$
\begin{aligned}
& \lim _{R \rightarrow+\infty}\left(T_{R}(t-\varepsilon)\left(\left(\alpha_{p}\left|T_{R}(\varepsilon) f\right|^{2}+\beta_{p} \eta_{R}^{2}\left|D T_{R}(\varepsilon) f\right|^{2}+\eta_{R}^{4}\left|D^{2} T_{R}(\varepsilon) f\right|^{2}\right)^{\frac{p}{2}}\right)\right)(x) \\
= & \left(T(t-\varepsilon)\left(\left(\alpha_{p}|T(\varepsilon) f|^{2}+\beta_{p}|D T(\varepsilon) f|^{2}+\left|D^{2} T(\varepsilon) f\right|^{2}\right)^{\frac{p}{2}}\right)\right)(x),
\end{aligned}
$$


uniformly for $s \in[\varepsilon, t-\varepsilon]$. Then, from (4.24) we get

$$
\begin{gathered}
\quad c_{p} \int_{\varepsilon}^{t-\varepsilon}\left\{T ( s ) \left[\left(\left|D \phi^{s}\right|^{2}+\left|D^{2} \phi^{s}\right|^{2}+\left|D^{3} \phi^{s}\right|^{2}\right)\right.\right. \\
\left.\left.\times\left(\alpha_{p}\left|\phi^{s}\right|^{2}+\beta_{p}\left|D \phi^{s}\right|^{2}+\left|D^{2} \phi^{s}\right|^{2}+\delta\right)^{\frac{p}{2}-1}\right]\right\}(x) d s \\
\leq\left(T(t-\varepsilon)\left(\alpha_{p}|T(\varepsilon) f|^{2}+\beta_{p}|D T(\varepsilon) f|^{2}+\left|D^{2} T(\varepsilon) f\right|^{2}\right)^{\frac{p}{2}}\right)(x),
\end{gathered}
$$

for any $x \in \mathbb{R}^{N}$. Now, from Lemma 4.2 it follows that

$$
\lim _{\varepsilon \rightarrow 0^{+}} D^{j} T(\varepsilon) f=D^{j} f, \quad j=0,1,2,
$$

uniformly in $B(k)$ for all $k>0$. Therefore from Lemma 4.6 we have

$$
\begin{aligned}
& \lim _{\substack{\varepsilon \rightarrow 0^{+}\\
}}\left(T(t-\varepsilon)\left(\alpha_{p}|T(\varepsilon) f|^{2}+\beta_{p}|D T(\varepsilon) f|^{2}+\left|D^{2} T(\varepsilon) f\right|^{2}\right)^{\frac{p}{2}}\right)(x) \\
= & \left(T(t)\left(\left(\alpha_{p}|f|^{2}+\beta_{p}|D f|^{2}+\left|D^{2} f\right|^{2}\right)^{\frac{p}{2}}\right)\right)(x),
\end{aligned}
$$

for any $x \in \mathbb{R}^{N}$. Hence, taking the limit as $\varepsilon$ tends to 0 in (4.25), we get

$$
\begin{aligned}
& c_{p} \int_{0}^{t} T(s)\left(\left(\left|D \phi^{s}\right|^{2}+\left|D^{2} \phi^{s}\right|^{2}+\left|D^{3} \phi^{s}\right|^{2}\right)\right. \\
& \left.\times\left(\alpha_{p}\left|\phi^{s}\right|^{2}+\beta_{p}\left|D \phi^{s}\right|^{2}+\left|D^{2} \phi^{s}\right|^{2}+\delta\right)^{\frac{p}{2}-1}\right)(x) d s \\
& \leq\left(T(t)\left(\alpha_{p} f^{2}+\beta_{p}|D f|^{2}+\left|D^{2} f\right|^{2}\right)^{\frac{p}{2}}\right)(x),
\end{aligned}
$$

for any $x \in \mathbb{R}^{N}$.

We now adapt the technique in [4, Proposition 4.3] to our situation. Let us observe that from (4.9) (with $l=2$ ) and the Young and Hölder inequalities, we deduce that for any $\gamma \in \mathbb{R}$,

$$
\begin{aligned}
& \left(|D T(t) f|^{2}+\left|D^{2} T(t) f\right|^{2}+\left|D^{3} T(t) f\right|^{2}\right)^{\frac{p}{2}} \\
= & \left(\left|D T(s)\left(\phi^{s}\right)\right|^{2}+\left|D^{2} T(s)\left(\phi^{s}\right)\right|^{2}+\left|D^{3} T(s)\left(\phi^{s}\right)\right|^{2}\right)^{\frac{p}{2}} \\
\leq & e^{\sigma_{3, p} s} T(s)\left[\left(\left|D \phi^{s}\right|^{2}+\left|D^{2} \phi^{s}\right|^{2}+\left|D^{3} \phi^{s}\right|^{2}\right)^{\frac{p}{2}}\right]+e^{\sigma_{3, p} s} T(s)\left(\left|\phi^{s}\right|^{p}\right) \\
\leq & e^{\sigma_{3, p} s} T(s)\left[\left(\left|D \phi^{s}\right|^{2}+\left|D^{2} \phi^{s}\right|^{2}+\left|D^{3} \phi^{s}\right|^{2}\right)^{\frac{p}{2}}\right. \\
& \times\left(\alpha_{p}\left|\phi^{s}\right|^{2}+\beta_{p}\left|D \phi^{s}\right|^{2}+\left|D^{2} \phi^{s}\right|^{2}+\delta\right)^{-\gamma} \\
& \left.\times\left(\alpha_{p}\left|\phi^{s}\right|^{2}+\beta_{p}\left|D \phi^{s}\right|^{2}+\left|D^{2} \phi^{s}\right|^{2}+\delta\right)^{\gamma}\right] \\
& +e^{\sigma_{3, p} s} T(t)\left(|f|^{p}\right) \quad e^{\sigma_{3, p} s}\left\{T ( s ) \left[\left(\left|D \phi^{s}\right|^{2}+\left|D^{2} \phi^{s}\right|^{2}+\left|D^{3} \phi^{s}\right|^{2}\right)\right.\right. \\
\quad & \left.\left.\times\left(\alpha_{p}\left|\phi^{s}\right|^{2}+\beta_{p}\left|D \phi^{s}\right|^{2}+\left|D^{2} \phi^{s}\right|^{2}+\delta\right)^{-\frac{2 \gamma}{p}}\right]\right\}^{\frac{p}{2}} \\
& \times\left\{T(s)\left[\left(\alpha_{p}\left|\phi^{s}\right|^{2}+\beta_{p}\left|D \phi^{s}\right|^{2}+\left|D^{2} \phi^{s}\right|^{2}+\delta\right)^{\frac{2 \gamma}{2-p}}\right]\right\}^{1-\frac{p}{2}} \\
+ & e^{\sigma_{3, p} s} T(t)\left(|f|^{p}\right) .
\end{aligned}
$$


Choosing $\gamma=p(2-p) / 4$, using Jensen and Young inequalities, and recalling that

$$
\begin{aligned}
& \left(\left|\phi^{s}\right|^{2}+\left|D \phi^{s}\right|^{2}+\left|D^{2} \phi^{s}\right|^{2}+\delta\right)^{\frac{p}{2}} \\
\leq & e^{\sigma_{2, p}(t-s)} T(t-s)\left(\left(f^{2}+|D f|^{2}+\left|D^{2} f\right|^{2}+\delta\right)^{\frac{p}{2}}\right)+\delta^{\frac{p}{2}}
\end{aligned}
$$

(see again (4.8)), we get for all $\varepsilon>0$

$$
\begin{gathered}
\left(|D T(t) f|^{2}+\left|D^{2} T(t) f\right|^{2}+\left|D^{3} T(t) f\right|^{2}\right)^{\frac{p}{2}} \\
\leq e^{\sigma_{3, p} s}\left\{T ( s ) \left[\left(\left|D \phi^{s}\right|^{2}+\left|D^{2} \phi^{s}\right|^{2}+\left|D^{3} \phi^{s}\right|^{2}\right)\right.\right. \\
\left.\left.\times\left(\alpha_{p}\left|\phi^{s}\right|^{2}+\beta_{p}\left|D \phi^{s}\right|^{2}+\left|D^{2} \phi^{s}\right|^{2}+\delta\right)^{\frac{p}{2}-1}\right]\right\}^{\frac{p}{2}} \\
\times\left\{T(s)\left[\left(\alpha_{p}\left|\phi^{s}\right|^{2}+\beta_{p}\left|D \phi^{s}\right|^{2}+\left|D^{2} \phi^{s}\right|^{2}+\delta\right)^{\frac{p}{2}}\right]\right\}^{1-\frac{p}{2}}+e^{\sigma_{3, p} s} T(t)\left(|f|^{p}\right) \\
\leq e^{\sigma_{3, p} s}\left\{\frac { p } { 2 } \varepsilon ^ { \frac { 2 } { p } } T ( s ) \left[\left(\left|D \phi^{s}\right|^{2}+\left|D^{2} \phi^{s}\right|^{2}+\left|D^{3} \phi^{s}\right|^{2}\right)\right.\right. \\
\left.\times\left(\alpha_{p}\left|\phi^{s}\right|^{2}+\beta_{p}\left|D \phi^{s}\right|^{2}+\left|D^{2} \phi^{s}\right|^{2}+\delta\right)^{\frac{p}{2}-1}\right] \\
\left.\left.+e^{\sigma_{3, p} s} T(t)\left(|f|^{p}\right) \quad \frac{p}{2}\right) \varepsilon^{\frac{2}{p-2}} T(s)\left[\left(\alpha_{p}\left|\phi^{s}\right|^{2}+\beta_{p}\left|D \phi^{s}\right|^{2}+\left|D^{2} \phi^{s}\right|^{2}+\delta\right)^{\frac{p}{2}}\right]\right\} \\
\leq e^{\sigma_{3, p} s}\left\{\frac { p } { 2 } \varepsilon ^ { \frac { 2 } { p } } T ( s ) \left[\left(\left|D \phi^{s}\right|^{2}+\left|D^{2} \phi^{s}\right|^{2}+\left|D^{3} \phi^{s}\right|^{2}\right)\right.\right. \\
\left.\times\left(\alpha_{p}\left|\phi^{s}\right|^{2}+\beta_{p}\left|D \phi^{s}\right|^{2}+\left|D^{2} \phi^{s}\right|^{2}+\delta\right)^{\frac{p}{2}-1}\right] \\
+\left(1-\frac{p}{2}\right) \varepsilon^{\frac{2}{p-2}} \max \left\{\alpha_{p}, \beta_{p}, 1\right\}^{\frac{p}{2}} \\
\times\left[e^{\sigma_{2, p}(t-s)} T(t)\left(\left(f^{2}+|D f|^{2}+\left|D^{2} f\right|^{2}+\delta\right)^{\frac{p}{2}}\right)+\delta^{\frac{p}{2}}\right] \\
\left.+T(t)\left(|f|^{p}\right)\right\} .
\end{gathered}
$$

Multiplying the first and last side of (4.28) by $e^{-\sigma_{3, p} s}$, integrating in $(0, t)$, pointwise with respect to $x \in \mathbb{R}^{N}$, and taking (4.27) into account, we get

$$
\begin{aligned}
& \frac{1-e^{-\sigma_{3, p} t}}{\sigma_{3, p}}\left(|D T(t) f|^{2}+\left|D^{2} T(t) f\right|^{2}+\left|D^{3} T(t) f\right|^{2}\right)^{\frac{p}{2}} \\
& \leq \frac{p}{2 c_{p}} \varepsilon^{\frac{2}{p}} T(t)\left(\left(\alpha_{p} f^{2}+\beta_{p}|D f|^{2}+\left|D^{2} f\right|^{2}\right)^{\frac{p}{2}}\right)+t T(t)\left(|f|^{p}\right) \\
&+\left(1-\frac{p}{2}\right) \varepsilon^{\frac{2}{p-2}} \max \left\{\alpha_{p}, \beta_{p}, 1\right\}^{\frac{p}{2}} \\
& \quad \times\left[\frac{e^{\sigma_{2, p} t}-1}{\sigma_{2, p}} T(t)\left(\left(f^{2}+|D f|^{2}+\left|D^{2} f\right|^{2}+\delta\right)^{\frac{p}{2}}\right)+\delta^{\frac{p}{2}}\right] .
\end{aligned}
$$


Letting $\delta$ tend to 0 in (4.29) gives

$$
\begin{aligned}
& \frac{1-e^{-\sigma_{3, p} t}}{\sigma_{3, p}}\left(|D T(t) f|^{2}+\left|D^{2} T(t) f\right|^{2}+\left|D^{3} T(t) f\right|^{2}\right)^{\frac{p}{2}} \\
\leq & \max \left\{\alpha_{p}, \beta_{p}, 1\right\}^{\frac{p}{2}}\left\{\min _{\varepsilon>0}\left(\frac{p}{2 c_{p}} \varepsilon^{\frac{2}{p}}+\left(1-\frac{p}{2}\right) \frac{e^{\sigma_{2, p} t}-1}{\sigma_{2, p}} \varepsilon^{\frac{2}{p-2}}\right)+t\right\} \\
\times & T(t)\left(\left(f^{2}+|D f|^{2}+\left|D^{2} f\right|^{2}\right)^{\frac{p}{2}}\right) \\
= & \max \left\{\alpha_{p}, \beta_{p}, 1\right\}^{\frac{p}{2}}\left[c_{p}^{-\frac{p}{2}}\left(\frac{e^{\sigma_{2, p} t}-1}{\sigma_{2, p}}\right)^{1-\frac{p}{2}}+t\right] T(t)\left(\left(f^{2}+|D f|^{2}+\left|D^{2} f\right|^{2}\right)^{\frac{p}{2}}\right)
\end{aligned}
$$

and (4.16) follows with $p \in(1,2)$.

The case when $p=2$ is similar and even simpler. It suffices to apply the previous arguments to the function $g_{0}$ defined by (4.17) (with $\delta=0$ and $p=2$ ).

In the case when $p>2$, (4.16) (with $l=3$ ) can be obtained from the case when $p=2$ observing that

$$
\begin{aligned}
\left|\left(D^{3} T(t) f\right)(x)\right|^{p} & =\left(\left|\left(D^{3} T(t) f\right)(x)\right|^{2}\right)^{\frac{p}{2}} \\
& \leq\left(\frac{\sigma_{3,2}}{\left(1-e^{-\sigma_{3,2} t}\right)} \phi_{3,2}(t)\right)^{\frac{p}{2}}\left(\left(T(t)\left(f^{2}+|D f|^{2}+\left|D^{2} f\right|^{2}\right)\right)(x)\right)^{\frac{p}{2}}
\end{aligned}
$$

and then applying Hölder's inequality.

The proof of (4.16) in the case when $l=1,2$ is completely similar, hence it is omitted. Actually, one can prove that

$$
\begin{aligned}
& \left(\sum_{j=0}^{l}\left|\left(D^{j} T(t) f\right)(x)\right|^{2}\right)^{\frac{p}{2}} \\
\leq & \left(\frac{\sigma_{l, p \wedge 2}}{1-e^{-\sigma_{l, p \wedge 2} t}} \phi_{l, p \wedge 2}(t)\right)^{\max \left\{1, \frac{p}{2}\right\}}\left(T(t)\left(\sum_{j=0}^{l-1}\left|D^{j} f\right|^{2}\right)^{\frac{p}{2}}\right)(x),
\end{aligned}
$$

for any $t>0, x \in \mathbb{R}^{N}, p \in(1,+\infty)$.

Corollary 4.8. Let $l \in\{2,3\}$ and let assumptions H1-H3, H4-l- $\gamma$ be satisfied. Then, for any $f \in C_{b}\left(\mathbb{R}^{N}\right)$ and any $p \in(1,+\infty)$ we have

$$
\left|\left(D^{l} T(t) f\right)(x)\right|^{p} \leq\left(\sum_{k=1}^{l} \prod_{j=0}^{k-1} \frac{\hat{\sigma}_{l-j, p \wedge 2}}{1-e^{-\hat{\sigma}_{l-j, p \wedge 2} t / l}} \phi_{l-j, p \wedge 2}(t / l)\right)^{\max \left\{1, \frac{p}{2}\right\}} \quad\left(T(t)\left(|f|^{p}\right)\right)(x),
$$

for any $t>0$ and any $x \in \mathbb{R}^{N}$, where $\phi_{j, r}$ and $\hat{\sigma}_{j, r}$ are as in Theorem 4.7 .

Proof. We begin by proving (4.31) in the case when $l=3$ and $p \leq 2$. We fix $f \in C_{b}\left(\mathbb{R}^{N}\right)$ and $t>0$. Splitting $T(t) f=T(t / 3) T(2 t / 3) f$ and applying (4.16) with $f$ being replaced by $T(2 t / 3) f$, we get

$$
\begin{aligned}
\left|\left(D^{3} T(t) f\right)(x)\right|^{p} \leq & \frac{\hat{\sigma}_{3, p}}{1-e^{-\hat{\sigma}_{3, p} t / 3}} \phi_{3, p}(t / 3) \\
& \times T(t / 3)\left\{\left(|T(2 t / 3) f|^{2}+|D T(2 t / 3) f|^{2}+\left|D^{2} T(2 t / 3) f\right|^{2}\right)^{\frac{p}{2}}\right\}(x),
\end{aligned}
$$


for any $x \in \mathbb{R}^{N}$. Now, taking (4.30) into account, with $T(t) f$ replaced by $T(2 t / 3) f$, we get

$$
\begin{aligned}
& \left(|T(2 t / 3) f|^{2}+|D T(2 t / 3) f|^{2}+\left|D^{2} T(2 t / 3) f\right|^{2}\right)^{\frac{p}{2}} \\
& \leq \frac{\hat{\sigma}_{2, p}}{1-e^{-\hat{\sigma}_{2, p} t / 3}} \phi_{2, p}(t / 3) T(t / 3)\left\{\left(|T(t / 3) f|^{2}+|D T(t / 3) f|^{2}\right)^{\frac{p}{2}}\right\}+T(l t / 3)\left(|f|^{p}\right) \\
& \leq \frac{\hat{\sigma}_{2, p}}{1-e^{-\hat{\sigma}_{2, p} t / 3}} \phi_{2, p}(t / 3)\left[\frac{\hat{\sigma}_{1, p}}{1-e^{-\hat{\sigma}_{1, p} t / 3}} \phi_{1, p}(t / 3) T(2 t / 3)\left(|f|^{p}+T(2 t / 3)\left(|f|^{p}\right)\right]\right. \\
& \quad+T(2 t / 3)\left(|f|^{p}\right) .
\end{aligned}
$$

Combining (4.32) and (4.33) gives (4.31) for $p \leq 2$. If $p>2$ and $l=3$, 4.31) follows from the case when $p=2$ applying Hölder's inequality. The proof of (4.31) (with $l=2)$ is similar: we write $\left|\left(D^{2} T(t) f\right)(x)\right|^{2}=\left|\left(D^{2} T(t / 2) T(t / 2) f\right)(x)\right|^{2}$ and apply (4.16), respectively with $l=1$ and $l=2$, to $T(t / 2) f$. We omit the details.

We now prove a Liouville-type theorem. Namely, we show that under suitable assumptions on the coefficients, if $u \in C_{b}\left(\mathbb{R}^{N}\right)$ satisfies the equation $\mathcal{A} u=0$, then $u$ is constant. As already stressed in the Introduction, such a result fails in general. We refer the reader to [18] for further details.

Theorem 4.9. Suppose that assumptions H1-H3 and H4-1- $\gamma(\gamma \in(0,1))$ hold true. Further assume that

$$
\frac{C^{2} N^{3} \nu_{0}^{\gamma-1}}{4}+M_{1}<0
$$

where $M_{1}$ is given by (4.1) and $C$ is as in $H_{4}-1-\gamma$. If $f \in C_{b}\left(\mathbb{R}^{N}\right)$ satisfies $\mathcal{A} f=0$, then $f$ is constant.

Proof. We observe that if $f \in C_{b}\left(\mathbb{R}^{N}\right)$ is such that $\mathcal{A} f=0$, then, by local elliptic regularity, it follows that $f \in D_{\max }(\mathcal{A})$ (see (3.35)). Moreover, the function $t \mapsto(T(t) f)(x)$ is continuously differentiable in $(0,+\infty)$ and $D_{t}((T(t) f)(x))=$ $(\mathcal{A} T(t) f)(x)=(T(t) \mathcal{A} f)(x)$ (see e.g. [12, Theorem 4.1]). Hence, $D_{t} T(t) f=0$ for any $t>0$, so that $T(t) f=f$ for any $t>0$. From (4.16) we deduce that

$$
|(D T(t) f)(x)| \leq \frac{\widetilde{C}}{\sqrt{t}} e^{\omega t}\left(\left(T(t) f^{2}\right)(x)\right)^{1 / 2} \leq \frac{\widetilde{C}}{\sqrt{t}} e^{\omega t}\|f\|_{\infty}, \quad \forall t>0, x \in \mathbb{R}^{N},
$$

for some $\widetilde{C}>0$ and some $\omega<0$. Indeed, (4.34) implies that $\hat{\sigma}_{1,2}=0$. As $t$ tends to $+\infty$, we get

$$
D f(x)=\lim _{t \rightarrow+\infty}(D T(t) f)(x)=0, \quad \forall x \in \mathbb{R}^{N},
$$

so that $f$ is constant.

4.1. Further estimates for $\mathcal{A}=\Delta+\sum_{j=1}^{N} b_{j}(x) D_{j}$. We now consider the particular case when $q_{i j}=\delta_{i j}$. It is well known that in this case the gradient estimate (4.2) (with $l=1$ ) can also be proved for $p=1$. More precisely, if there exists a constant $d_{0} \in \mathbb{R}$ such that $\sum_{i, j=1}^{N} D_{i} b_{j}(x) \xi_{i} \xi_{j} \leq d_{0}|\xi|^{2}$ for any $x, \xi \in \mathbb{R}^{N}$, then we have

$$
|(D T(t) f)(x)| \leq e^{d_{0} t} T(t)(|D f|)(x), \quad \forall t>0, x \in \mathbb{R}^{N},
$$

for any $f \in C_{b}^{1}\left(\mathbb{R}^{N}\right)$ (see e.g. [1, 2, 4]). 
With this in mind, here we extend the estimates for the second- and third-order derivatives given in Theorem 4.7 to the case $p=1$.

Theorem 4.10. Let $l \in\{2,3\}$ and let assumptions H2, H4-l- $\gamma\left(\right.$ with $\left.q_{i j}=\delta_{i j}\right)$ and (3.4) be satisfied. Then,

$$
\left|\left(D^{l} T(t) f\right)(x)\right| \leq e^{\sigma_{l} t}\left(T(t)\left[\left(\sum_{j=1}^{l}\left|D^{j} f\right|^{2}\right)^{\frac{1}{2}}\right](x)\right), \quad \forall t>0, x \in \mathbb{R}^{N},
$$

where $\sigma_{l}=M_{l}+(l-1) M_{l}^{+}($see (4.1) $)$.

Proof. We limit ourselves to sketching the proof in the case when $l=2$.

Let $\delta>0, f \in C_{b}^{1}\left(\mathbb{R}^{N}\right)$ and consider the function $w_{\delta}: \mathbb{R}_{+} \times \mathbb{R}^{N} \rightarrow \mathbb{R}$ defined by

$$
w_{\delta}(t, x)=\left(|D u(t, x)|^{2}+\left|D^{2} u(t, x)\right|^{2}+\delta\right)^{\frac{1}{2}},
$$

where, as usual, $u(t, \cdot)=T(t) f$. Arguing as in the proof of Theorem 4.3 and taking Cauchy-Schwarz inequality into account, we can easily show that $w_{\delta}$ turns out to solve the Cauchy problem

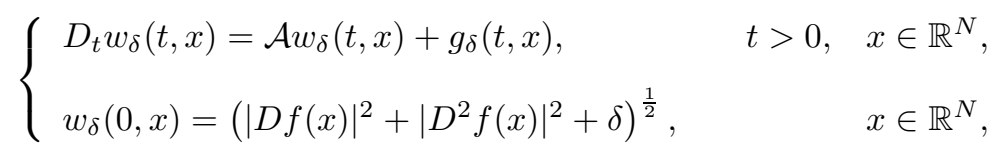

where

$g_{\delta} \leq w_{\delta}^{-1}\left(\sum_{j, h=1}^{N} D_{h} b_{j} D_{j} u D_{h} u+2 \sum_{j, h, k=1}^{N} D_{h} b_{j} D_{j k} u D_{h k} u+\sum_{j, h, k=1}^{N} D_{h k} b_{j} D_{j} u D_{h k} u\right)$.

From $H_{4}-2-\gamma$ we get for all $\varepsilon>0$

$$
g_{\delta} \leq w_{\delta}^{-1}\left(\left(d+\frac{N^{2}}{4 \varepsilon} r\right)|D u|^{2}+2\left(d+\frac{\varepsilon N}{2} r\right)\left|D^{2} u\right|^{2}\right) .
$$

We choose $\varepsilon=\sqrt{N / 2}$ so that $\varepsilon N / 2=N^{2} /(4 \varepsilon)=N^{3 / 2} / \sqrt{8}$ and we get

$$
d(x)+\frac{N^{2}}{4 \varepsilon} r(x) \leq M_{2}, \quad d(x)+\frac{\varepsilon N}{2} r(x) \leq M_{2}, \quad \forall x \in \mathbb{R}^{N} .
$$

Now, if $M_{2} \geq 0$ it follows that $g_{\delta} \leq 2 M_{2} w_{\delta}$; if $M_{2}<0$ we have

$$
g_{\delta} \leq M_{2}\left(|D u|^{2}+\left|D^{2} u\right|^{2}+\delta\right)^{-\frac{1}{2}}\left(|D u|^{2}+\left|D^{2} u\right|^{2}\right) \leq M_{2} w_{\delta}-M_{2} \delta^{\frac{1}{2}} .
$$

Following step by step the proofs of Theorems 4.3 and 4.4 we can easily get (4.35).

Remark 4.11. It is well known that in general estimates (4.16) and (4.31) fail for $p=1$. We refer the reader to [4, Example 5.5] where a counterexample is exhibited in the case when $\mathcal{A}=\Delta$.

\section{INVARIANT MEASURES}

In this section we will show that the pointwise estimates of the previous section imply global estimates for the derivatives of $T(t) f$ in $L^{p}$-spaces associated with the semigroup invariant measure, whenever it exists. 
We recall that a probability measure $\mu$ in $\mathbb{R}^{N}$ is an invariant measure of the semigroup $\{T(t)\}_{t \geq 0}$ if

$$
\int_{\mathbb{R}^{N}}(T(t) f)(x) d \mu(x)=\int_{\mathbb{R}^{N}} f(x) d \mu(x),
$$

for any $f \in C_{b}\left(\mathbb{R}^{N}\right)$ and any $t>0$. If an invariant measure exists, then it is unique, it is absolutely continuous with respect to the Lebesgue measure and it has a strictly positive density (see for example [13, Section 6]).

The Has'minskii theorem shows that if there exists a nonnegative function $\varphi \in$ $C^{2}\left(\mathbb{R}^{N}\right)$ such that $\lim _{|x| \rightarrow+\infty} \mathcal{A} \varphi(x)=-\infty$, then there exists an invariant measure of the semigroup $\{T(t)\}_{t \geq 0}$. See [8, Chapter III, Theorem 5.1] for more details.

If an invariant measure $\mu$ exists, then the semigroup $\{T(t)\}_{t \geq 0}$ can be extended to a strongly continuous contraction semigroup in $L_{\mu}^{p}$, which, in general, is not analytic (see [14]).

The following theorem both extends the results in [4, Corollary 5.4] to our situation, and gives, for any $p \in(1,+\infty), L^{p}$-estimates for the second- and third-order derivatives of $T(t)$ under the same assumptions on the coefficients as in Section 4 For this purpose, we observe that estimates (4.16) (with $l=1$ ) and (4.31) can be rewritten in a simpler and more readable way as

$$
\left|\left(D^{j} T(t) f\right)(x)\right|^{p} \leq C_{j, p} \frac{e^{\omega_{j, p} t}}{t^{j / 2}}\left(T(t)|f|^{p}\right)(x), \quad \forall t>0, x \in \mathbb{R}^{N},
$$

for any $p \in(1,+\infty)$, any $j=1,2,3$ and some constants $C_{j, p}>0$ and $\omega_{j, p} \in \mathbb{R}$.

Theorem 5.1. Let $\mu$ be the invariant measure associated with the semigroup $T(t)$. Moreover, let $l \in\{1,2,3\}$ and let assumptions H1-H3, $\mathrm{H}_{4}-\mathrm{l}-\gamma$ be satisfied, and fix $p \in(1,+\infty)$. Then, if $\omega_{j, p} \leq 0$,

$$
\left\|D^{l} T(t) f\right\|_{p} \leq C_{l, p} \frac{e^{\omega_{l, p} t}}{t^{l / 2}}\|f\|_{p}, \quad \forall t>0 .
$$

If $\omega_{l, p}>0$, then for any $\omega>0$ there exist positive constants $\widetilde{C}_{l, p}=\widetilde{C}_{l, p}(\omega)$ such that

$$
\left\|D^{l} T(t) f\right\|_{p} \leq \widetilde{C}_{l, p} \frac{e^{\omega t}}{t^{l / 2}}\|f\|_{p}
$$

for any $f \in L^{p}\left(\mathbb{R}^{N}, \mu\right)$. In particular, if $l=1$, then $D\left(A_{p}\right) \subset W_{\mu}^{1, p}\left(\mathbb{R}^{N}\right)$ and there exists a positive constant $M=M(\omega)$ such that

$$
\|D u\|_{p} \leq M\|u\|_{p}^{\frac{1}{2}}\left\|\left(A_{p}-\omega\right) u\right\|_{p}^{\frac{1}{2}}
$$

Proof. We fix $f \in C_{b}\left(\mathbb{R}^{N}\right)$ and, for any fixed $t>0$, we integrate (5.1) in $\mathbb{R}^{N}$ obtaining

$$
\begin{aligned}
\int_{\mathbb{R}^{N}}\left|\left(D^{j} T(t) f\right)(x)\right|^{p} d \mu(x) & \leq C_{l, p} \frac{e^{\omega_{l, p} t}}{t^{l / 2}} \int_{\mathbb{R}^{N}}\left(T(t)\left(|f|^{p}\right)\right)(x) d \mu(x) \\
& =C_{l, p} \frac{e^{\omega_{l, p} t}}{t^{l / 2}} \int_{\mathbb{R}^{N}}|f(x)|^{p} d \mu(x) .
\end{aligned}
$$

By density we can extend (5.5) to all $f \in L_{\mu}^{p}$. If $\omega_{l, p} \leq 0$ we are done. In the case when $\omega_{l, p}>0$, from (5.5) we easily get (5.3) if we recall that $T(t)$ is a semigroup of contractions in $L_{\mu}^{p}$ and argue as in the last part of the proof of Theorem 3.3 .

Finally, we observe that (5.4) follows from (5.2) and (5.3) (with $l=1$ ), arguing as in the proof of [4, Corollary 5.4]. 


\section{REFERENCES}

[1] D. Bakry, Transformations de Riesz pour les semigroupes symétriques. Seconde partie: Etude sous la condition $\Gamma_{2} \geq 0$, Séminaire de probabilités XIX, pp. 145-174, Lect. Notes Math. 1123, Springer-Verlag, Berlin, 1985. MR0889473 (89h:42023)

[2] D. Bakry, M. Ledoux, Lévy-Gromov's isoperimetric inequality for an infinite dimensional diffusion generator, Invent. Math. 123 (1996), pp. 259-281. MR1374200 (97c:58162)

[3] M. Bertoldi, Analytic methods for Markov semigroups, Ph.D. thesis, Università di Trento, 2002.

[4] M. Bertoldi, S. Fornaro, Gradient estimates in parabolic problems with unbounded coefficients, Studia Math. 165 (2004), pp. 221-254. MR.2109509

[5] S. Cerrai, Second Order PDE's in Finite and Infinite Dimension, Lect. Notes Math. 1762, Springer-Verlag, Berlin, 2001. MR1840644 (2002j:35327)

[6] G. Da Prato, Regularity results for some degenerate parabolic equation, Rivista Mat. Univ. Parma 6 (1999), pp. 245-257. MR1752802 (2001c:35123)

[7] A. Friedman, Partial differential equations of parabolic type, Prentice Hall, Englewood Cliffs, N.J., 1964. MR0181836 (31:6062)

[8] R.Z. Has'minskii, Stochastic stability of differential equations, Nauka 1969 (in Russian), English translation: Sijthoff and Noordhoff 1980. MR0600653 (82b:60064)

[9] N.V. Krylov, Introduction to the theory of diffusion processes, American Mathematical Society, Providence, 1995. MR1311478 (96k:60196)

[10] O.A. Ladizhenskaja, V.A. Solonnikov, N.N. Ural'ceva, Linear and quasilinear equations of parabolic type, Nauka, English transl.: American Mathematical Society, Providence, 1968. MR $0241822(39: 3159 b)$

[11] A. Lunardi, Analytic Semigroups and Optimal Regularity in Parabolic Problems, Birkhäuser, Basel, 1995. MR 1329547|(96e:47039)

[12] A. Lunardi, Schauder theorems for linear elliptic and parabolic problems with unbounded coefficients in $\mathbb{R}^{N}$, Studia Math. 128 (1998), no. 2, pp. 171-198. MR1490820 (99a:35020)

[13] G. Metafune, D. Pallara, M. Wacker, Feller semigroups on $\mathbb{R}^{N}$, Semigroup Forum 65 (2002), pp. 159-205. MR.1911723 (2003i:35170)

[14] G. Metafune, E. Priola, Some classes of non-analytic Markov semigroups, J. Math. Anal. Appl. 294 (2004), 596-613. MR2061345

[15] G. Metafune, J. Prüss, A. Rhandi, R. Schnaubelt, The domain of the Ornstein-Uhlenbeck operator on an $L^{p}$ spaces with an invariant measure, Ann. Sc. Norm. Sup. Pisa Cl. Sci. 5, Vol. I (2002), pp. 471-485. MR1991148|(2004e:35040)

[16] G. Metafune, J. Prüss, A. Rhandi, R. Schnaubelt, $L^{p}$ regularity for elliptic operators with unbounded coefficients, report 21 Institute of Analysis Martin Luther, Universitaet Halle Wittenberg FB Mathematik und Informatik (2002).

[17] E. Priola, The Cauchy problem for a class of Markov-type semigroups, Commun. Appl. Anal. 5 (2001), no. 1, pp. 49-75. MR.1844671 (2003c:47077)

[18] E. Priola, J. Zabczyk, Liouville theorems for non local operators, J. Funct. Anal. 216 (2004), no. 2, pp. 455-490. MR2095690

[19] F.Y. Wang, On estimation of the logarithmic Sobolev constant and gradient estimates of heat semigroups, Probab. Theory Related Fields 108 (1997), pp. 87-101. MR1452551(98h:58184)

Applied Mathematical Analysis, Delft University of Technology, P.O. Box 5031, 2600 GA Delft, The Netherlands

E-mail address: bertoldi@fastmail.fm

Dipartimento di Matematica, Università di Parma, Via M. D'Azeglio 85/A, 43100 PARMA, ITALY

E-mail address: luca.lorenzi@unipr.it 